\title{
ESO imaging survey: optical deep public survey ${ }^{\star} \star \star \star$
}

\author{
A. Mignano ${ }^{1,2,3}$, J.-M. Miralles ${ }^{2,4}$, L. da Costa ${ }^{2,5}$, L. F. Olsen ${ }^{6,2,7}$, I. Prandoni ${ }^{3}$, S. Arnouts ${ }^{8}$, C. Benoist ${ }^{6}$, R. Madejsky ${ }^{9}$, \\ R. Slijkhuis ${ }^{2}$, and S. Zaggia ${ }^{10}$
}

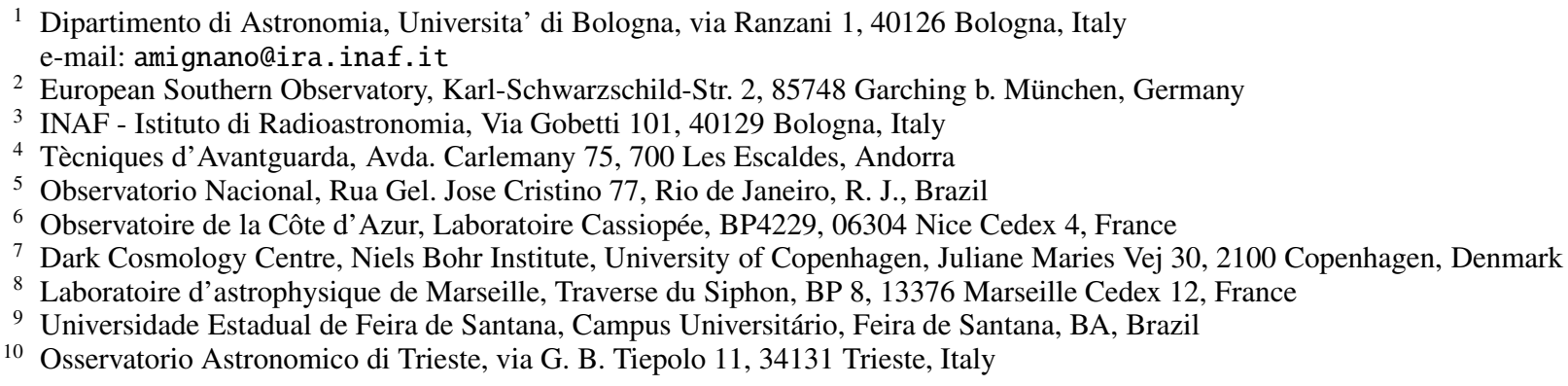

Received 16 February 2006 / Accepted 19 October 2006

\section{ABSTRACT}

\begin{abstract}
This paper presents new five passbands $(U B V R I)$ optical wide-field imaging data accumulated as part of the DEEP Public Survey (DPS) carried out as a public survey by the ESO Imaging Survey (EIS) project. Out of the 3 square degrees originally proposed, the survey covers 2.75 square degrees, in at least one band (normally $R$ ), and 1.00 square degrees in five passbands. The median seeing, as measured in the final stacked images, is $0{ }^{\prime} 97$, ranging from $0 \prime^{\prime} 75$ to $2^{\prime \prime} 0$. The median limiting magnitudes (AB system, $2^{\prime \prime}$ aperture, $5 \sigma$ detection limit) are $U_{A B}=25.65, B_{A B}=25.54, V_{A B}=25.18, R_{A B}=24.8$ and $I_{A B}=24.12$ mag, consistent with those proposed in the original survey design. The paper describes the observations and data reduction using the EIS Data Reduction System and its associated EIS/MVM library. The quality of the individual images were inspected, bad images discarded and the remaining used to produce final image stacks in each passband, from which sources have been extracted. Finally, the scientific quality of these final images and associated catalogs was assessed qualitatively by visual inspection and quantitatively by comparison of statistical measures derived from these data with those of other authors as well as model predictions, and from direct comparison with the results obtained from the reduction of the same dataset using an independent (hands-on) software system. Finally to illustrate one application of this survey, the results of a preliminary effort to identify sub-mJy radio sources are reported. To the limiting magnitude reached in the $R$ and $I$ passbands the success rate ranges from 66 to $81 \%$ (depending on the fields). These data are publicly available at CDS ${ }^{\star \star \star}$.
\end{abstract}

Key words. catalogs - surveys - stars: general - galaxy: general - radio continuum: general

\section{Introduction}

Deep multi-wavelength observations are a pre-condition for the selection of suitable galaxy samples for spectroscopic followup observation using the multiplex capability of spectrographs mounted on large-aperture telescopes. Foreseeing the need for such samples for the scientific exploitation of multi-object spectrographs such as FORS and VIMOS, ESO's Survey Working Group (SWG) recommended the ESO Imaging Survey (EIS, Renzini \& da Costa 1997) project to undertake a series of deep, optical/infrared observations. Based on ideas submitted by the ESO community and evaluated by the SWG, the DEEP Public Survey (DPS) was designed to provide a data set that could be used to study the large-scale distribution of galaxies at $z \sim 1$ and to identify a large number of Ly- $\alpha$ galaxies and, combined with

* Based on observations carried out at the European Southern Observatory, La Silla, Chile under program Nos. 164.O-0561, 169.A0725, and 267.A-5729.

$\star \star$ Appendices A, B and C are only available in electronic form at http://www . aanda. org

$\star \star \star$ Available at CDS via anonymous ftp to

cdsarc.u-strasbg.fr (130.79.128.5) or via

http://cdsweb.u-strasbg.fr/cgi-bin/qcat?J/A+A/462/553 near-infrared data, extremely red objects (EROs) for follow-up work using VLT. In its entirety this survey comprises three optical and infrared strategies. The optical part, the focus of the present paper, consists of a deep survey covering three regions of 1 square degree on the sky, in the $U-, B-, V-, R$ - and $I$ passbands, using the wide-field imager (WFI) mounted on the ESO/MPG $2.2 \mathrm{~m}$ telescope at La Silla. Each of the three DEEP regions $1,2,3$ - is covered by four WFI pointings (in five passbands) $\mathrm{a}, \mathrm{b}, \mathrm{c}, \mathrm{d}$. The regions were selected both to enable observations year-round and because they overlapped with regions of other scientific interest. For instance, DEEP1 was chosen to complement the sub-mJy ATESP radio survey (Prandoni et al. 2000a,b; Prandoni et al. 2006) carried out with the Australia Telescope Compact Array (ATCA) covering the region surveyed by the ESO Slice Project (Vettolani et al. 1997), while DEEP2 included the CDF-S field. Finally, DEEP3, was chosen in the northern galactic hemisphere, thus providing an almost year-round coverage $^{1}$. The location and the characteristics of the surveyed regions as well as the planned limiting magnitudes in each passband

1 The text for the original proposal can be found at http://www . eso.org/science/eis/documents.html 
can be found in the DPS strategy page $\mathrm{e}^{2}$ available in the EIS web-pages.

This paper is part of a series describing the data releases made by the EIS project. In Dietrich et al. (2006) (hereafter Paper I) the data reduction system and the general procedures adopted are described. Here the observations, reduction, and science verification of the optical data taken as part of the DPS, publicly released late 2004, are reviewed. Furthermore, in this paper changes to the dataset that have occurred since the original release, stemming from additional evaluation of the data, are also described. Complementing the optical results reported in the present paper, the results obtained from infrared observations covering these fields are presented in Olsen et al. (2006). Section 2 describes optical observations while the reduction and calibration of the data are presented in Sect. 3. Final survey products such as stacked images and science-grade catalogs extracted from them are presented in Sect. 4. The quality of these products is evaluated in Sect. 5 by comparing statistical measures obtained from these data with those of other authors. In this section the results of a preliminary assessment of the optical identification of sub-mJy radio sources from the ATESP $1.4 \mathrm{GHz}$ radio survey are also discussed. Finally, in Sect. 6 a brief summary of the paper is presented.

\section{Observations}

The optical observations were carried out using WFI at the ESO/MPG-2.2 m telescope. WFI is a focal reducer-type mosaic camera mounted at the Cassegrain focus of the telescope. The mosaic consists of $4 \times 2$ CCD chips with $2048 \times 4096$ pixels with a projected pixel size of 0 '.238, giving a FOV of 8 .'12 $\times 16^{\prime} .25$ for each individual chip. The chips are separated by gaps of $233^{\prime \prime} 8$ and 14 ". 3 along the right ascension and declination direction, respectively. The full FOV of WFI is thus $34^{\prime} \times 33^{\prime}$ with a filling factor of $95.9 \%$.

The data presented in this paper were obtained as part of the ESO Large Programme: 164.O-0561, carried out in visitor mode and its service mode continuation (30 h) 169.A-0725 (Principal Investigator: J. Krautter, as chair of the SWG) as well as the service mode program 267.A-5729.

Observations, nearly all in visitor mode, were performed in the $U-, B-, V-, R-$, and $I$-passbands. These were split into OBs consisting of a sequence of five (ten in the $I$-band) dithered subexposures with the typical exposure time given in Table 1. Filter curves can be found in Arnouts et al. (2001) and on the web page of the La Silla Science Operations Team ${ }^{3}$. Note that in an attempt to improve the performance of the survey, new $U$ and $I$ filters were purchased and used during the course of the observations. In addition, there were also changes in the observing strategy in the $U$-, to conform to the one hour limit for a single OB imposed by the telescope team, and the $I$-band, to improve the fringing correction. It is important to point out that the changes in strategy were unrelated to the change of the actual filter used.

The data from the above programs were accumulated in observations covering six semesters (ESO periods 64-69) from November 4, 1999 through September 28, 2002, corresponding to 1905 science exposures, and $246 \mathrm{~h}$ of on-target integration, during 76 nights. With the data presented in this paper $90 \%$ of the data accumulated by the DPS program have been

\footnotetext{
2 http://www.eso.org/science/eis/surveys/ strategy_DPS.html

${ }^{3}$ http://www.ls.eso.org/lasilla/sciops/2p2/E2p2M/ WFI/filters
}

Table 1. Typical OBs used in the observations for the DPS optical survey. The table gives: in Col. 1 the passband; in Col. 2 the filter id adopting the unique naming convention of the La Silla Science Operations Team; in Col. 3 the number of exposures in the OB; in Col. 4 the integration time of the individual sub-exposures in the $\mathrm{OB}$; and in Col. 5 the total exposure time in seconds.

\begin{tabular}{lcccc}
\hline \hline Passband & Filter & \# exposures & $\begin{array}{c}T_{\exp } \\
(\mathrm{s})\end{array}$ & $\begin{array}{c}T_{\text {tot }} \\
(\mathrm{s})\end{array}$ \\
\hline$U$ & $\# 877$ & 5 & 900 & 4500 \\
$U$ & $\# 841$ & 5 & 550 & 2750 \\
$B$ & $\# 842$ & 5 & 300 & 1500 \\
$V$ & $\# 843$ & 5 & 300 & 1500 \\
$R$ & $\# 844$ & 5 & 300 & 1500 \\
$I$ & $\# 879$ & 5 & 600 & 3000 \\
$I$ & $\# 845$ & 10 & 300 & 3000 \\
\hline
\end{tabular}

reduced and publicly released. The remaining 196 exposures include tests, wrong pointings, as well as discarded images. About $10 \%$ of the images in all filters have been discarded, with the exception of the $B$-band. The data covers 2.75 squares degrees, in at least one band, and 1.00 square degrees in five passbands.

Standard star observations are available for 75 out of the 76 nights with science observations. These consist of a total of roughly 500 standard star OBs, nearly all consisting of two images per OB. While this amounts to slightly more than $10 \mathrm{~h}$ ontarget (or $4 \%$ of the science observations in terms of time), it exceeds the volume of science frames. For three nights either no standard fields were observed (September 27, 2002) or they are not available for a particular filter (November 29, 2000, $U$-band and June 7, 2002, I-band). Depending on the night, the number of measurements can range from a few to over 300, covering from 1 to 3 Landolt fields (Landolt 1992).

\section{Data reduction}

The WFI data were reduced and calibrated using the EIS data reduction system and its associated EIS/MVM image processing library version 1.0 .1 . The latter is designed to automatically process images from single/multi-chip optical/infrared cameras. In addition to the standard bias-subtraction, flatfield correction and trimming, the package includes sophisticated algorithms for background estimation, de-fringing, astrometric calibration, minimization of chip-to-chip variations in sensitivity and detection/masking of satellite tracks so common in wide-field imagers. This software package is publicly available and can be retrieved from the EIS release page ${ }^{4}$. Details about the EIS/MVM library can be found in Vandame (2004). In Paper I the reduction and general handling of WFI surveys through the EIS data reduction system is described. In the following the points specific for the DPS WFI data are described.

The astrometric calibration was derived using the GSC 2.2 reference catalog and a distortion model described by a second order polynomial. Comparisons with independent astrometric catalogs yield a typical scatter of 0.2 arcsec (Vandame 2004). However, comparisons made using exposures of a globular cluster, shifted by half a field, strongly suggest that the internal accuracy is about 70 mas. The astrometric calibration is carried out on a chip by chip basis, which occasionally may lead to less than optimal PSF as discussed in Paper I, which also describes possible improvements to be implemented to the code in the future. The reduced images are warped onto a user-defined

\footnotetext{
${ }^{4}$ http://www.eso.org/science/eis/survey_release.html
} 
Table 2. Number of nights with different types of photometric solution. The table gives in Col. 1 the passband; in Col. 2 the filter identification; in Col. 3 the number of default solutions (corresponding to the number of non-photometric nights and/or nights without standard star observations); in Cols. 4-6 the number of nights with 1-, 2- or 3-parameter fits, respectively; and in Col. 7 the total number of nights in which standard star observations were carried out with a given filter.

\begin{tabular}{ccccccc}
\hline \hline Passband & Filter id & Default & 1-par & 2-par & 3-par & Total \\
\hline$U$ & $\# 877$ & 3 & 17 & 12 & 6 & 38 \\
$U$ & $\# 841$ & 0 & 9 & 0 & 0 & 9 \\
$B$ & $\# 842$ & 0 & 13 & 2 & 2 & 17 \\
$V$ & $\# 843$ & 0 & 10 & 7 & 6 & 23 \\
$R$ & $\# 844$ & 5 & 6 & 6 & 2 & 19 \\
$I$ & $\# 879$ & 1 & 0 & 2 & 1 & 4 \\
$I$ & $\# 845$ & 6 & 9 & 4 & 7 & 26 \\
\hline
\end{tabular}

Table 3. Type of photometric solution per passband for each observed DPS field. The table gives: in Col. 1 the region; in Col. 2 the field; in Cols. 3-6 the type of best solution available for a given passband.

\begin{tabular}{|c|c|c|c|c|c|}
\hline Region & Field name & Default & 1-par & 2-par & 3-par \\
\hline DEEP1 & DEEP1a & & $U B$ & $I$ & $U R$ \\
\hline DEEP1 & DEEP1b & & $B$ & $U$ & $V R I$ \\
\hline DEEP1 & DEEP1c & & & $V R$ & \\
\hline DEEP2 & DEEP2a & & & $R$ & \\
\hline DEEP2 & DEEP2b & & $B$ & $U$ & $V R I$ \\
\hline DEEP2 & DEEP2c & & $U I$ & $U$ & $V$ \\
\hline DEEP2 & DEEP2d & & $R$ & & \\
\hline DEEP3 & DEEP3a & & $U U R I$ & & $B V$ \\
\hline DEEP3 & DEEP3b & & & $R$ & $U B V I$ \\
\hline DEEP3 & DEEP3c & & & $B V R$ & $U I$ \\
\hline DEEP3 & DEEP3d & & $B$ & V & $I$ \\
\hline
\end{tabular}

reference grid (pixel, projection and orientation), using a thirdorder Lanczos kernel. The WCS in the image header is reported using the CD-matrix notation.

The EIS Data Reduction System also includes a photometric pipeline for automatic extraction of the photometric solutions used to calibrate reduced images as described in detail in Paper I. As mentioned above, standard stars were observed in 75 nights corresponding to about 500 OBs. These were used to obtain photometric solutions for each night for calibrating the reduced images.

The EIS reduction system derives a number of photometric solutions for each night. Depending on the airmass and color coverage between one and three parameters are fit. Among these solutions the one with the smallest scatter is considered the best fit and refered to as the "best solution". Table 2 summarizes the number of nights with standard star observations and type of "best solution" obtained by the photometric pipeline for each passband. It can be seen that for all filters, except $U \# 841,2$ - and 3 -parameter fits are available, in principle, allowing for reliable photometric calibration. In the case of the $U \# 841$ the airmass coverage was insufficient and only one-parameter fits are available. Neither does the filter have a solution from the Telescope Team thus hampering further confirmation.

Table 3 gives the type of fit for the "best solution" obtained for each field and filter indicating the quality of the photometric calibration. It can be seen that for all field/filter combinations there is at least one reduced image calibrated with at least a 1-parameter fit. In addition, with exception of the $B$-band data for DEEP1 and DEEP2, all others have at least a 2-parameter fit solution, thereby ensuring a reasonable photometric calibration. More importantly, the table also shows that in nearly all regions
Table 4. Median values for all photometric solutions based on 3-parameter fits. The table lists: in Col. 1 the passband/filter; in Cols. 24 the median zeropoint in Vega System $(Z p)$, extinction, $(k)$, and color term (color) values for all 3-parameter fit solutions.

\begin{tabular}{cccc}
\hline \hline Passband & $Z p$ & $k$ & color \\
\hline$U \# 877$ & 22.06 & 0.5 & 0.04 \\
$B \# 842$ & 24.58 & 0.22 & 0.24 \\
$V \# 843$ & 24.23 & 0.19 & -0.04 \\
$R \# 844$ & 24.49 & 0.08 & -0.01 \\
$I \# 879$ & 23.35 & 0.02 & 0.03 \\
$I \# 845$ & 23.18 & 0.09 & 0.24 \\
\hline
\end{tabular}

Table 5. Comparison between EIS and Telescope Team solutions. The table lists: in Col. 1 the passband; in Cols. 2-4 the offsets (EISTelescope Team) of the zeropoint, extinction and color term.

\begin{tabular}{cccc}
\hline \hline Passband & $Z p$ & $k$ & color \\
\hline$U \# 877$ & 0.0 & 0.02 & -0.01 \\
$V \# 843$ & 0.08 & 0.08 & 0.09 \\
$R \# 844$ & 0.02 & 0.01 & -0.01 \\
I\#879 & -0.02 & 0.02 & 0.0 \\
\hline
\end{tabular}

a 3-parameter fit exists for at least one field thus allowing the photometric calibration to be bootstrapped or cross-checked to other fields.

As part of the photometric calibration, nights with good airmass and color coverage were used to compute complete photometric solutions, namely those for which all parameters (zeropoint, extinction and color term) can be estimated. The median values derived for these 3-parameter fits are shown in Table 4. As mentioned earlier, no such solution is available for the $U$-band filter \#841.

The photometric solutions computed automatically by the EIS data reduction system were compared with the "best solution" recently obtained by the $2 \mathrm{p} 2$ Telescope Team. The results of this comparison are presented in Table 5 for the general case of 3-parameter fits. The solutions are remarkably similar, despite the fact that they are over two years apart. It is worth emphasizing that, for the present release, the periods of observations of standard stars available to the two teams do not coincide. Also note that there are three (in $U \# 841, B \# 842^{5}$ and $I \# 845$ ) filters EIS has used for which no solutions have been reported by the telescope team. These cases are not shown in the table.

The quality of the photometric calibration of the present dataset can further be assessed from Table 6. Considering only 3 -parameter fit solutions and after rejecting 3 sigma outliers one finds that the scatter of the zeropoints is less than about $0.1 \mathrm{mag}$, which is a reasonable estimate for the accuracy of the absolute photometric calibration of the DPS survey data. Not surprisingly, larger offsets are found when 2- and 1-parameter fits are included, depending on the passband and estimator used for extinction and color term. One sees that considerable work remains to be done to significantly reduce the photometric calibration error which is typically of the order of $0.1 \mathrm{mag}$. A notable exception is the wider $U \# 877$, for which the rms exceeds 0.2 mag, indicating that it will be difficult to obtain a proper calibration for this filter.

As final points regarding the photometric calibration of the DPS data, it is important to emphasize that the CCD-toCCD gain variations have been corrected using the median

\footnotetext{
5 The solution for the $B$-band reported in the web-page does not correspond the same filter as used here.
} 
Table 6. Average parameters for all photometric solutions and constraining the sample to the 3-parameter fits. The table gives: in Col. 1 the passband/filter combination; in Col. 2 the median zeropoint in Vega System $(Z p)$ including solutions obtained from fits with an arbitrary number of free parameters; in Col. 3 the scatter computed from the distribution of zeropoints (rms); in Col. 4 the largest offset of a night calibration relative to the median reported, $(\Delta Z p)_{\max }$; in Cols. 5-7 the same as in the three previous columns, except that now the estimate of the median is computed using only 3-parameter fits.

\begin{tabular}{ccccccc}
\hline \hline Passband & $Z p$ & rms & $(\Delta Z p)_{\max }$ & $Z p$ & rms & $(\Delta Z p)_{\max }$ \\
\hline U\#877 & 22.02 & 0.27 & -0.72 & 22.06 & 0.22 & -0.68 \\
U\#841 & 21.58 & 0.08 & 0.11 & - & - & - \\
B\#842 & 24.64 & 0.08 & -0.21 & 24.58 & 0.05 & -0.27 \\
V\#843 & 24.18 & 0.08 & 0.16 & 24.23 & 0.1 & 0.21 \\
R\#844 & 24.52 & 0.08 & -0.17 & 24.49 & 0.09 & -0.2 \\
I\#879 & 23.34 & 0.02 & 0.02 & 23.35 & 0.0 & 0.03 \\
I\#845 & 23.14 & 0.11 & -0.3 & 23.18 & 0.13 & -0.26 \\
\hline
\end{tabular}

background values sampled in sub-regions bordering adjacent CCDs but, as in Paper I, no illumination correction has been applied which may lead to relative magnitude offsets from the center to the borders of the image.

The EIS/MVM library was used to convert the available 1905 raw WFI science exposures $(171 \mathrm{~Gb})$ into 331 fully calibrated reduced images, of which 304 were released and are discussed in the present paper. Of the remaining 27 , one was observed with the narrow-band $I$ filter \# 854 and 26 (1 $U, 6 \mathrm{~B}$, $6 V, 1 R$ and $13 I$ ) images were rejected during visual inspection (grading). Therefore, the present paper considers 304 fully calibrated, reduced WFI images in the following passbands: 1) $118 U$-band images - 21 using filter \#841, with a mean wavelength of $3676 \AA(F W H M=325 \AA)$, and 97 using the wider filter \#877, central wavelength of $3404 \AA(F W H M=732 \AA)$; 2) $38 B$-band images with a mean wavelength of $4562 \AA(F W H M=$ $990 \AA$ ); 3 ) $35 V$-band images with a mean wavelength of $5395 \AA$ $(F W H M=893 \AA)$; 4) $48 R$-band images with a mean wavelength of $6517 \AA(F W H M=1621 \AA)$; and 5) 65 I-band images - 8 obtained using the $I$ filter \#879, with a mean wavelength of $8269 \AA$ (5 nights in the period from March 10, 2002 to June 7, 2002), and 57 using the filter \#845 with a central wavelength of $8643 \AA$, and $F W H M=1387 \AA$ (25 nights in the period from November 4, 1999 to August 21, 2001). The available data covers 11 fields of the 12 requested. The completeness of the observations is listed in Table 11 for each stacked image and the corresponding "completeness" plots are accessible from the EIS web-pages. The zero point of each of the reduced images was computed based on the photometric solution of the night of observations. If no solution could be obtained, either because of missing data or due to a non-photometric night, a default zero point was assigned as detailed in Paper I.

Before being released all the science images were examined by eye and graded from A (best) to D (worst). Out of 331 reduced images covering the selected DPS fields, $142(43 \%)$ were graded A, $121(37 \%) B, 41(12 \%) \mathrm{C}$ and $26(8 \%) \mathrm{D}$. The grade distribution is a function of the passband as can be seen from Table 7 showing the grade breakdown for each filter. The table shows that for $B V R$ bands over $75 \%$ of the products are graded $\mathrm{A}$, while for $U$-band the typical grade is $B$ and in $I$ band all grades are roughly equally represented. In the $U$-band images (both filters), the low grades are mainly due to clearly visible electronic noise because of the low level counts of the background in most exposures (550-900 s). In the $I$-band the
Table 7. Distribution of grades per passband. The table gives: in Col. 1 the passband; in Col. 2 the ESO filter number; in Col. 3 the number of images; in Cols. 4-7 the ratio of images in a given grade to the total number of images taken with that filter.

\begin{tabular}{ccccccc}
\hline \hline Passband & Filter & \# reduced & $\mathrm{A}$ & $\mathrm{B}$ & $\mathrm{C}$ & $\mathrm{D}$ \\
\hline$U$ & $\# 877$ & 97 & 0.22 & 0.69 & 0.09 & 0.0 \\
$U$ & $\# 841$ & 22 & 0.05 & 0.91 & 0.00 & 0.05 \\
$B$ & $\# 842$ & 44 & 0.82 & 0.02 & 0.02 & 0.14 \\
$V$ & $\# 843$ & 41 & 0.73 & 0.02 & 0.1 & 0.15 \\
$R$ & $\# 844$ & 48 & 0.77 & 0.19 & 0.04 & 0.0 \\
$I$ & $\# 879$ & 8 & 0.63 & 0.25 & 0.13 & 0.0 \\
$I$ & $\# 845$ & 70 & 0.17 & 0.3 & 0.34 & 0.19 \\
\hline
\end{tabular}

low grades are usually caused by residual fringing. The latter is especially true in the case of filter \#845 due to the long integration time used per exposure, leading to inadequate estimate of the fringing map. Specific details about the fringing removal procedure of EIS/MVM can be found in Vandame (2004). Even though some reduced images have grades $C$ they are still useful in building the final stack, therefore only images with grade D are rejected. These $\mathrm{C}$ graded images may present a higher photometric noise but the estimated variations in flux are less than $10 \%$. A graphic display of the grade distribution can be accessed from the release page of this survey in the EIS web-pages. Note that images with grade D were not released, since they have no scientific value, and were discarded from the stacking process discussed below.

It is important to emphasize that the grade attributed to a product depends on the stage of the process. In fact, the grade of the reduced images does not reflect the quality of the final "stacked" image, which in general have considerably better grades (see below). For instance, the reduced images still show a number of cosmic ray hits, because the construction of reduction blocks (RB) is optimized for removing cosmic ray features in the final stacks. In most cases, this implies that each field and filter is observed with at least $3 \mathrm{RBs}$. Therefore, in most cases the stack blocks (SB) consist of at least 3 input frames, allowing for the use of a thresholding procedure to remove cosmic ray hits from the final stacked image. In contrast to Paper I, over $60 \%$ of the reduction blocks consist of 5 or less frames. This has the effect of leaving the imprint of the inter-chip gaps, leading to a larger number of cosmic rays in the reduced images and in the final stack at the location of the inter-chip gaps (see Paper I). As in the case of Paper I the masking of satellite tracks seemed to have worked remarkably well.

\section{Final products}

\subsection{Images}

Final stacked images for each observed DPS field were produced from nightly reduced images properly grouped in Stacking Blocks (SBs) as explained in detail in Paper I. The 304 reduced images with grades better than D were converted into 42 stacked (co-added) images out of which 40 were released and described in the present paper. These stacks and their associated logs are publicly available in the EIS web pages. Figure 1 shows a color composite image of the DEEP1b field typical for the fields observed in this survey. These high-galactic latitude fields contrast with those of Paper I, which also included a number of lowgalactic latitude fields.

As before all images were visually inspected and graded. Out of the 42 stacked images covering the DPS fields, 30 were graded 


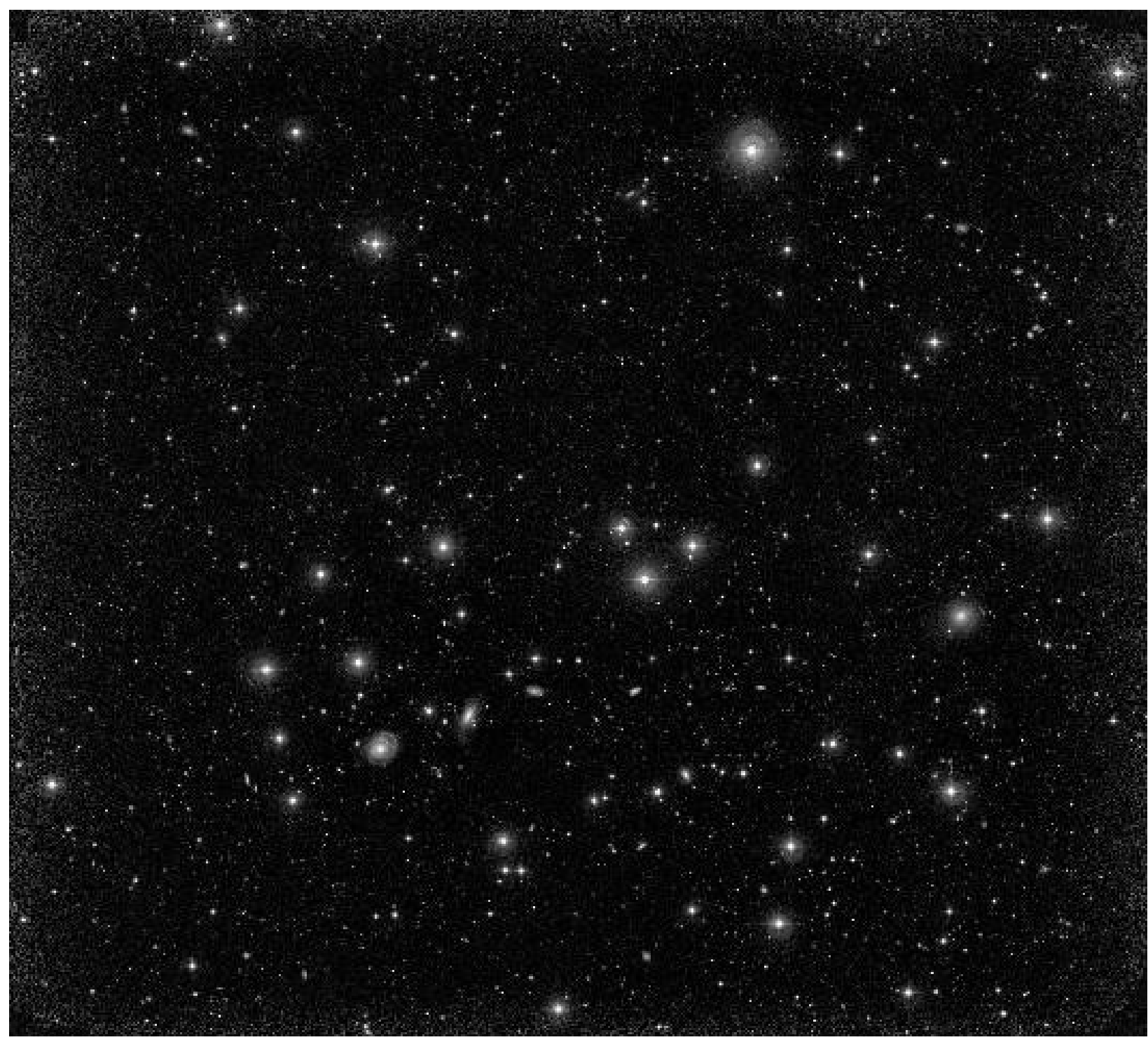

Fig. 1. Color composite $B V R$-image of the DEEP1b field with north up and east to the left. The image covers the entire WFI field of $34^{\prime} \times 33^{\prime}$. It also demonstrates the accuracy of the astrometric calibration independently achieved in each passband.

A, $6 \mathrm{~B}, 4 \mathrm{C}$, and $2 \mathrm{D}$. When appropriate a comment is associated to the image. Table 8 illustrates that by listing the 21 images for which comments were made, regardless of their grade.

Of the two images graded "D", one, the $B$-band image of DEEP3d, was discarded because it was a single image of 5 minutes exposure time, and the other, an I-band image of DEEP3a produced from a single RB comprising 6 exposures, because it had a very strong fringing pattern.

Table 9 shows for each filter the grade breakdown for the stacked images. Not surprisingly, comparison with Table 7 shows that, in general, the grades of stacks are considerably better than those of the reduced images. In particular, in contrast to the reduced images, the $U$-bands are mostly graded A. The notable exception are the $I$-band (\#845) images, of which a significant fraction are graded $\mathrm{C}$, in fact all images graded $\mathrm{C}$ are $I$-band, for which the procedure of de-fringing was less than ideal. This is in marked contrast with the results reported in Paper I for the XMM survey, indicating the importance of the observing strategy for properly correcting for fringing. The results also show that fringing is the single most important problem faceed by WFI multi-wavelength surveys.
The accuracy of the final photometric calibration depends on the accuracy of the photometric calibration of the reduced images which are used to produce the final co-added stacks and the number of independent photometric nights in which these were observed. The former depends not only on the quality of the night but also on the adopted calibration plan. To preview the quality of the photometric calibration, Table 10 provides information on the number of reduced images and number of independent nights for each passband and filter. Examining this table together with Table 3 provides some insight into the quality of the photometric calibration of each final stack.

The main attributes of the stacks produced for each field/filter combination are given in Table 11. The table can also be used to assess the completeness of the survey as a whole.

To provide a backbone infrastructure to allow the user to have an overview of the quality of the released data, an integral part of the data release infrastructure provided by the EIS Data Reduction System is to produce figures illustrating the distribution of attributes of the released products. These distributions for the data considered are made available in the EIS web pages. as part of the data release. The figures include: the distribution of 
Table 8. Grades and comments for stacked images with an associated comment. The table, ordered by increasing grade, lists: in Col. 1 the field name; in Col. 2 the passband; in Col. 3 the grade; and in Col. 4 the associated comment.

\begin{tabular}{lccl}
\hline \hline Field name & Passband & Grade & Comment \\
\hline DEEP3a & $I \# 845$ & D & fringing \\
DEEP3d & $B \# 842$ & D & single 300 s image \\
DEEP1a & $I \# 845$ & C & fringing \\
DEEP2b & $I \# 845$ & C & fringing \\
DEEP2c & $I \# 845$ & C & fringing \\
DEEP3c & $I \# 845$ & C & fringing - Poor background subtraction near bright stars \\
DEEP1a & $U \# 841$ & B & visible electronic noise \\
DEEP1b & $U \# 877$ & B & visible electronic noise \\
DEEP2c & $U \# 841$ & B & visible electronic noise \\
DEEP3a & $U \# 841$ & B & visible electronic noise \\
DEEP3a & $U \# 877$ & B & visible electronic noise \\
DEEP2d & $R \# 844$ & B & Reflections, few images stacked \\
DEEP3b & $I \# 845$ & B & fringing near bright star in lower left (SE) \\
DEEP1b & $I \# 845$ & A & low level fringing \\
DEEP2a & $R \# 844$ & A & poor background subtraction around the bright star on the lower left corner \\
DEEP2b & $V \# 843$ & A & residual traces of the inter-chip gaps visible \\
DEEP3a & $R \# 844$ & A & stray light reflections in the upper and lower left corners \\
DEEP3a & $V \# 843$ & A & stray light reflections in the upper and lower left corners \\
DEEP3a & $B \# 842$ & A & stray light reflections in the upper and lower left corners \\
DEEP3c & $R \# 844$ & A & poor background subtraction near bright stars \\
DEEP3d & $I \# 879$ & A & low level fringing - stray light reflection on the lower right corner \\
DEEP3d & $V \# 843$ & A & stray light reflection on the lower right corner \\
\hline
\end{tabular}

Table 9. Grades assigned to the DPS stacked images as a function of the passband. The table gives: in Col. 1 the passband; in Col. 2 the ESO filter number; in Col. 3 the total number of stacked images for each passband; in Cols. 4-7 the ratio of images with a given grade to the total number of images taken with that filter.

\begin{tabular}{ccccccc}
\hline \hline Passband & Filter & \#Stacks & $\mathrm{A}$ & $\mathrm{B}$ & $\mathrm{C}$ & $\mathrm{D}$ \\
\hline$U$ & $\# 877$ & 7 & 0.71 & 0.29 & 0.0 & 0.0 \\
$U$ & $\# 841$ & 3 & 0.33 & 0.67 & 0.0 & 0.0 \\
$B$ & $\# 842$ & 7 & 0.86 & 0.0 & 0.0 & 0.14 \\
$V$ & $\# 843$ & 8 & 1.0 & 0.0 & 0.0 & 0.0 \\
$R$ & $\# 844$ & 9 & 0.88 & 0.12 & 0.0 & 0.0 \\
$I$ & $\# 879$ & 1 & 1.0 & 0.0 & 0.0 & 0.0 \\
$I$ & $\# 845$ & 7 & 0.14 & 0.14 & 0.57 & 0.14 \\
\hline
\end{tabular}

grades, limiting magnitude (Vega, 5 sigma, $2^{\prime \prime}$ aperture), seeing and an estimate of the rms of the PSF distortions; and the relation of limiting magnitude (Vega, 5 sigma, 2" aperture) versus seeing and integration time. For the sake of space these plots are not reproduced here, even though the present release may differ slightly from that shown in the web.

\subsection{Source Catalogs}

Catalogs were extracted from the image stacks using the SExtractor program (version 2.3.2, Bertin \& Arnouts 1996) and a common configuration file, with the option of using the weightmap associated to each image. Details about the choice of input parameters and their fine-tuning can be found in Paper I.

Before being released the catalogs were superposed onto the images, examined by eye and graded. The grade is meant to be a subjective assessment of the overall appearance of the catalog in terms of spurious objects, automatic masking of satellite tracks and bright objects. To some extent the catalog grade reflects the quality of the image. Information regarding the scientific quality of the catalog can be found in the verification section of the associated product log. Out of the 40 catalogs released, 8 were graded $\mathrm{A}, 23 \mathrm{~B}, 7 \mathrm{C}$, and $2 \mathrm{D}$. In contrast to the images, the two catalogs graded D are also being released in order to illustrate the impact of inadequate de-fringing. It is important to note that the catalogs graded $\mathrm{D}$ were extracted from grade $\mathrm{C}$ images. Table 12 presents all 34 cases where comments were made.

In general, there is a correlation between the grade of the catalogs and the grade of the images from which they were extracted. However, the catalog grade can be lower than that of the associated image due to effects such as the detection of large number of spurious objects associated with the ghost images of bright stars, stray light, and others. For instance, all the $U$-band catalogs were graded B. As in the case of the corresponding images, the $I$-band catalogs are typically $\mathrm{C}$ and $\mathrm{D}$. The following two cases should be noted:

1. DEEP1a $(I)$ - the original image was graded $\mathrm{C}$ because of observed fringing in the final stack. This has led to a catalog with no scientific value because of the high number of spurious objects detected along the fringing pattern. This catalog is being released exclusively as an illustration.

2. DEEP2b $(I)$ - same as above

The inspection of the projected distribution of objects, strongly suggests that the automatic masking of satellite tracks has worked remarkably well as no prominent linear features, a signature of this type of problem, are seen on the inspected catalogs. A description of the catalog format is found in the appendices of Paper I.

\section{Discussion}

\subsection{Comparison of counts and colors}

A key element in public surveys is to provide potential users with information regarding the quality of the products released. To this end a number of checks of the data are carried out and several diagnostics plots summarizing the results are automatically produced by the EIS Survey System. They are an integral part of the product logs available from the survey release page. Due to the large number of plots produced in the verification 
Table 10. Number of reduced images and number of independent nights for each field and passband. The table gives for each field: in Col. 1 the region; in Col. 2 the field name; in Cols. 3-9 the number of reduced images, and in parenthesis, the number of independent nights for each passband.

\begin{tabular}{clccccccc}
\hline \hline Region & Field name & $U \# 877$ & $U \# 841$ & $B \# 842$ & $V \# 843$ & $R \# 844$ & $I \# 879$ & $I \# 845$ \\
\hline DEEP1 & DEEP1a & $20(12)$ & $5(2)$ & $4(1)$ & $0(0)$ & $4(2)$ & $0(0)$ & $10(4)$ \\
DEEP1 & DEEP1b & $11(9)$ & $0(0)$ & $6(4)$ & $5(5)$ & $11(4)$ & $0(0)$ & $11(6)$ \\
DEEP1 & DEEP1c & $0(0)$ & $0(0)$ & $0(0)$ & $3(3)$ & $6(4)$ & $0(0)$ & $0(0)$ \\
DEEP2 & DEEP2a & $0(0)$ & $0(0)$ & $0(0)$ & $0(0)$ & $6(3)$ & $0(0)$ & $0(0)$ \\
DEEP2 & DEEP2b & $17(5)$ & $0(0)$ & $7(2)$ & $5(2)$ & $7(3)$ & $0(0)$ & $8(5)$ \\
DEEP2 & DEEP2c & $12(5)$ & $9(3)$ & $0(0)$ & $7(3)$ & $0(0)$ & $0(0)$ & $12(3)$ \\
DEEP2 & DEEP2d & $0(0)$ & $0(0)$ & $0(0)$ & $0(0)$ & $2(2)$ & $0(0)$ & $0(0)$ \\
DEEP3 & DEEP3a & $10(4)$ & $7(4)$ & $9(6)$ & $5(5)$ & $6(5)$ & $0(0)$ & $1(1)$ \\
DEEP3 & DEEP3b & $9(3)$ & $0(0)$ & $6(2)$ & $4(2)$ & $2(1)$ & $0(0)$ & $7(5)$ \\
DEEP3 & DEEP3c & $18(7)$ & $0(0)$ & $5(2)$ & $3(2)$ & $4(3)$ & $0(0)$ & $8(6)$ \\
DEEP3 & DEEP3d & $0(0)$ & $0(0)$ & $1(1)$ & $3(2)$ & $0(0)$ & $8(5)$ & $0(0)$ \\
\hline
\end{tabular}

Table 11. Overview of the attributes of the produced image stacks and survey completeness. The table gives: in Col. 1 the field name; in Col. 2 the passband; in Col. 3 the filter identification; in Col. 4 the total integration time $T_{\text {int }}$ in seconds, of the final stack; in Col. 5 the number of contributing reduced images or RBs; in Col. 6 the total number of science frames contributing to the final stack; in Cols. 7 and 8 the seeing in arcseconds and the point-spread function (PSF) anisotropy measured in the final stack; in Col. 9 the limiting magnitude, $m_{\text {lim }}$, estimated for the final image stack for a $2^{\prime \prime}$ aperture, $5 \sigma$ detection limit in the Vega system; in Col. 10 the grade assigned to the final image during visual inspection (ranging from A to D); in Col. 11 the fraction (in percentage) of observing time relative to that originally planned.

\begin{tabular}{|c|c|c|c|c|c|c|c|c|c|c|}
\hline Field & Passband & Filter & $\begin{array}{r}T_{\text {int }} \\
(\mathrm{s}) \\
\end{array}$ & \#RBs & \#Еxp. & $\begin{array}{r}\text { Seeing } \\
(\operatorname{arcsec})\end{array}$ & PSF rms & $\begin{array}{r}m_{\lim } \\
\text { (Vega mag) }\end{array}$ & Grade & $\begin{array}{r}\text { Completeness } \\
(\%)\end{array}$ \\
\hline DEEP1a & $\bar{U}$ & \#877 & 77393 & 20 & 86 & 1.37 & 0.217 & 25.26 & $\mathrm{~A}$ & 179 \\
\hline DEEP1a & $U$ & \#841 & 18298 & 5 & 21 & 1.28 & 0.086 & 24.42 & B & 30 \\
\hline DEEP1a & $B$ & \#842 & 11397 & 4 & 38 & 1.37 & 0.139 & 25.85 & A & 90 \\
\hline DEEP1a & $R$ & \#844 & 9897 & 4 & 33 & 0.87 & 0.169 & 25.74 & A & 110 \\
\hline DEEP1a & $I$ & \#845 & 29693 & 10 & 83 & 0.86 & 0.217 & 23.76 & $\mathrm{C}$ & 110 \\
\hline DEEP1b & $\bar{U}$ & \#877 & 27546 & 11 & 45 & 1.17 & 0.235 & 24.62 & $\mathrm{~B}$ & 64 \\
\hline DEEP1b & $B$ & \#842 & 11397 & 6 & 38 & 1.43 & 0.139 & 25.66 & A & 90 \\
\hline DEEP1b & V & \#843 & 10497 & 5 & 35 & 1.31 & 0.231 & 25.35 & A & 117 \\
\hline DEEP1b & $R$ & \#844 & 20694 & 11 & 69 & 1.29 & 0.173 & 25.32 & A & 230 \\
\hline DEEP1b & $I$ & \#845 & 28492 & 11 & 95 & 0.97 & 0.229 & 24.19 & A & 106 \\
\hline DEEP1c & $\bar{V}$ & $\# 843$ & 7498 & 3 & 25 & 1.19 & 0.147 & 25.03 & $\bar{A}$ & 83 \\
\hline DEEP1c & $R$ & \#844 & 11997 & 6 & 40 & 0.98 & 0.147 & 25.43 & A & 133 \\
\hline DEEP2a & $R$ & $\# 844$ & 5998 & 6 & 20 & 0.84 & 0.105 & 24.51 & $\mathrm{~A}$ & 67 \\
\hline DEEP2b & $U$ & \#877 & 55795 & 15 & 62 & 1.2 & 0.251 & 23.87 & $\mathrm{~A}$ & 129 \\
\hline DEEP $2 b$ & $B$ & \#842 & 11997 & 7 & 40 & 0.98 & 0.212 & 26.48 & A & 95 \\
\hline DEEP $2 b$ & V & \#843 & 9297 & 5 & 31 & 0.89 & 0.189 & 25.19 & A & 103 \\
\hline DEEP $2 b$ & $R$ & \#844 & 11997 & 7 & 40 & 1.36 & 0.127 & 25.06 & A & 133 \\
\hline DEEP2b & $I$ & \#845 & 17697 & 8 & 42 & 0.81 & 0.146 & 23.77 & $\mathrm{C}$ & 66 \\
\hline$\overline{D E E P} 2 \mathrm{c}$ & $\bar{U}$ & \#877 & 45896 & $\overline{12}$ & 51 & 2.26 & 0.265 & 25.05 & $\bar{A}$ & 106 \\
\hline DEEP2c & $U$ & \#841 & 32397 & 9 & 36 & 0.94 & 0.332 & 24.56 & A & 53 \\
\hline DEEP2c & V & \#843 & 16597 & 7 & 39 & 1.07 & 0.218 & 24.94 & A & 184 \\
\hline DEEP2c & $I$ & \#845 & 29695 & 9 & 50 & 0.91 & 0.155 & 23.94 & $\mathrm{C}$ & 110 \\
\hline DEEP2d & $\bar{R}$ & $\# 844$ & 600 & 1 & 2 & 1.00 & 0.096 & 23.74 & $\bar{B}$ & 7 \\
\hline DEEP3a & $U$ & \#877 & 35997 & 10 & 40 & 1.12 & 0.223 & 24.29 & B & 83 \\
\hline DEEP3a & $U$ & \#841 & 27897 & 7 & 31 & 1.28 & 0.159 & 24.11 & B & 46 \\
\hline DEEP3a & $B$ & \#842 & 11997 & 9 & 40 & 0.99 & 0.178 & 27,73 & A & 95 \\
\hline DEEP3a & V & \#843 & 8997 & 5 & 30 & 1.03 & 0.171 & 25.1 & A & 100 \\
\hline DEEP3a & $R$ & \#844 & 9597 & 6 & 32 & 0.82 & 0.158 & 24.99 & A & 107 \\
\hline DEEP3a & $I$ & \#845 & 3599 & 1 & 6 & 1.50 & 0.047 & 22.3 & D & 13 \\
\hline DEEP3b & $U$ & \#877 & 35997 & 9 & 40 & 0.96 & 0.224 & 24.39 & $\bar{A}$ & 83 \\
\hline DEEP3b & $B$ & \#842 & 11697 & 6 & 39 & 0.96 & 0,211 & 27.2 & A & 93 \\
\hline DEEP3b & V & \#843 & 8997 & 4 & 30 & 0.89 & 0.157 & 26.68 & A & 100 \\
\hline DEEP3b & $R$ & \#844 & 5998 & 2 & 20 & 0.78 & 0.096 & 24.73 & A & 67 \\
\hline DEEP3b & $I$ & \#845 & 20995 & 7 & 65 & 0.84 & 0.155 & 23.35 & B & 78 \\
\hline$\overline{D E E P 3 c}$ & $\bar{U}$ & \#877 & 48844 & 18 & 64 & 0.99 & 0.266 & 23.89 & $\mathrm{~A}$ & 113 \\
\hline DEEP3c & $B$ & \#842 & 13496 & 5 & 45 & 0.93 & 0.182 & 25.83 & A & 107 \\
\hline DEEP3c & V & \#843 & 5998 & 3 & 20 & 0.79 & 0.121 & 25.07 & A & 67 \\
\hline DEEP3c & $R$ & \#844 & 8997 & 4 & 30 & 0.81 & 0.154 & 25.43 & A & 100 \\
\hline DEEP3c & $I$ & \#845 & 23993 & 8 & 80 & 0.98 & 0.182 & 23.85 & $\mathrm{C}$ & 89 \\
\hline DEEP3d & $\bar{B}$ & $\# 842$ & 300 & 1 & 1 & 1.58 & 0.072 & 25.32 & $\overline{\mathrm{D}}$ & 2 \\
\hline DEEP3d & V & \#843 & 5998 & 3 & 20 & 0.95 & 0.107 & 24.75 & A & 67 \\
\hline DEEP3d & $I$ & \#879 & 23193 & 8 & 79 & 0.78 & 0.178 & 24.32 & A & 86 \\
\hline
\end{tabular}


Table 12. Grades for source lists with associated comment. The table, ordered by increasing grade, lists: in Col. 1 the EIS field name; in Col. 2 the passband; in Col. 3 the grade; and in Col. 4 the associated comment.

\begin{tabular}{|c|c|c|c|}
\hline Field name & Passband & Grade & Comment \\
\hline DEEP1a & I\#845 & $\mathrm{D}$ & Fringing leads to multiple spurious detections \\
\hline DEEP $2 b$ & I\#845 & $\mathrm{D}$ & Fringing leads to multiple spurious detections \\
\hline DEEP2a & $R \# 844$ & $\mathrm{C}$ & $\begin{array}{l}\text { Large number of spurious objects caused by very bright star at the lower left } \\
\text { edge of the image. Missing masks around a few saturated stars }\end{array}$ \\
\hline DEEP2c & I\#845 & $\mathrm{C}$ & $\begin{array}{l}\text { Reflections from bright stars cause spurious objects in various areas of the image. } \\
\text { Low level fringing adds also additional noise in the catalog }\end{array}$ \\
\hline DEEP2d & R\#844 & $\mathrm{C}$ & $\begin{array}{l}\text { Numerous cosmic rays misidentified as real objects. Spurious objects caused by } \\
\text { reflection ring of bright star in the lower left quadrant }\end{array}$ \\
\hline DEEP3b & I\#845 & $\mathrm{C}$ & Multiple spurious detections at the lower left quadrant due to fringing \\
\hline $\mathrm{DEEP} 3 \mathrm{c}$ & R\#844 & $\mathrm{C}$ & $\begin{array}{l}\text { Missing masks for a few saturated stars. Multiple spurious objects caused by } \\
\text { the reflection rings of the bright stars at the top and bottom right of the image }\end{array}$ \\
\hline DEEP3c & I\#845 & $\mathrm{C}$ & $\begin{array}{l}\text { Spurious objects caused by fringing at the lower left quadrant of the image } \\
\text { Multiple spurious objects caused by the reflection rings of bright stars } \\
\text { at the top and bottom right of the image }\end{array}$ \\
\hline DEEP3d & I\#879 & $\mathrm{C}$ & Spurious objects caused by multiples reflection rings/stray light across the image \\
\hline DEEP1a & U\#877 & B & Spurious objects around bright stars \\
\hline DEEP1a & $U \# 841$ & B & Few spurious objects around bright stars \\
\hline DEEP1a & B\#842 & B & Mask missing for a few saturated stars \\
\hline DEEP1b & U\#877 & B & Masks missing around saturated stars. Spurious objects around bright stars \\
\hline DEEP1b & I\#845 & B & Residual fringing increases the number of spurious objects \\
\hline DEEP1c & $V \# 843$ & B & Masks missing around a few saturated stars \\
\hline DEEP1c & R\#844 & B & Masks missing around a few saturated stars \\
\hline DEEP2b & U\#877 & B & Masks missing around all saturated stars \\
\hline $\mathrm{DEEP} 2 \mathrm{~b}$ & $B \# 842$ & B & $\begin{array}{l}\text { Spurious objects caused by reflections of bright star at center top of the image. } \\
\text { Missing masks for several saturated stars }\end{array}$ \\
\hline DEEP2b & $V \# 843$ & B & $\begin{array}{l}\text { Spurious objects caused by reflections of bright stars at the top center } \\
\text { and lower right corner of the image }\end{array}$ \\
\hline DEEP2c & U\#877 & B & Missing masks for a few saturated stars \\
\hline DEEP2c & $U \# 841$ & B & Missing masks around a few saturated stars \\
\hline DEEP3a & U\#877 & B & $\begin{array}{l}\text { Spurious objects around bright stars. Spurious objects caused by stray light } \\
\text { at the top left corner }\end{array}$ \\
\hline DEEP3a & U\#841 & B & $\begin{array}{l}\text { Spurious objects around bright stars. Spurious objects caused by stray light } \\
\text { at the top left corner }\end{array}$ \\
\hline DEEP3a & B\#842 & B & $\begin{array}{l}\text { Masks missing for a few saturated stars. Spurious objects caused by stray light } \\
\text { at the top left corner }\end{array}$ \\
\hline DEEP3a & $V \# 843$ & B & $\begin{array}{l}\text { Masks missing for a few saturated stars. Spurious objects caused by stray light } \\
\text { at the top left corner }\end{array}$ \\
\hline DEEP3a & $R \# 844$ & B & $\begin{array}{l}\text { Masks missing for a few saturated stars. Spurious objects caused by stray light } \\
\text { at the top and bottom left corners }\end{array}$ \\
\hline DEEP3b & U\#877 & B & Spurious objects around bright stars \\
\hline DEEP3b & B\#842 & B & Missing masks around a few saturated stars \\
\hline DEEP3b & $V \# 843$ & B & Masks missing around a few saturated stars \\
\hline DEEP3b & R\#844 & B & Spurious objects caused by left-over cosmic rays at the inter-chip gaps \\
\hline DEEP3c & U\#877 & B & Spurious objects around bright stars \\
\hline DEEP3c & V\#843 & B & $\begin{array}{l}\text { Multiple spurious objects caused by the reflection ring of the bright stars } \\
\text { at the top and bottom right of the image }\end{array}$ \\
\hline DEEP1b & $R \# 844$ & A & Mask missing around 1 saturated star at the center-right of the image \\
\hline DEEP2b & R\#844 & A & Few spurious objects at the lower right corner caused by bright star reflection at the edge of the image \\
\hline
\end{tabular}

process these are not reproduced here. Instead, to illustrate the results only a small set of these plots are shown.

A relatively simple statistics that can be used to check the catalogs and the star/galaxy separation criteria is to compare the star and galaxy number counts derived from the data to that of other authors and/or to model predictions. For stars we use Vega magnitudes and for galaxies $\mathrm{AB}$ magnitudes, while for both we use the MAG_AUTO magnitudes from the catalog. Figures A.1-A.3 in Appendix A show for each region a mosaic where the rows represent the passbands from $U$ (top) to $I$ (bottom) and the columns the different fields available from a (left) to d (right). Here objects with CLASS_STAR $<0.95$ were considered to be galaxies. Note that the number counts shown in the figure take into account the effective covered area, which is available in the FIELDS table (see Appendix D of Paper I). Inspection of all figures shows that the galaxy counts obtained from the final produced stacks are, in general, in excellent agreement with those obtained by previous authors, (Arnouts et al. 2001; Metcalfe et al. 2001), regardless of the passband. The figures also highlight some of the challenges in dealing with mosaics, as the completeness of the counts can be seen to vary from field to field.

A complementary test is to compare the stellar counts to those predicted by models such as the galactic model of Girardi et al. (2005). Figures B.1-B.3 in Appendix B show these comparisons, in the same format as the galaxy counts, for each of the observed regions. The good agreement between the data and the model predictions is indeed remarkable, especially considering 
the uncertainties in the zero-point and the model. These results give further support to the photometric calibration and the scientific quality of the science-grade data produced automatically by the EIS system.

While useful to detect gross errors, number counts are not sufficiently sensitive to identify more subtle differences. The comparison of expected colors of stars with theoretical models provides a better test of the accuracy of the photometric calibration in the different bands. Using the model of Girardi et al. (2005), the predicted colors of stars can be obtained and compared to the data to evaluate at least the rough consistency of the photometric calibration in different passbands. Such comparisons were made for each of the four fields covered in all five passbands and are shown in Figs. C.1-C.4 presented in Appendix C. From these figures one finds that at least for the fields with complete color information there is a fairly good agreement between the locus of stars and those predicted by the model in four different color-color diagrams, indicating a good ( $\lesssim 0.1 \mathrm{mag}$ ) consistency among the photometric calibration obtained in different passbands.

\subsection{Comparison with other reductions}

The data presented here have been reduced using the same method and parameter settings as those discussed in Paper I. In that paper the results of the reduction through the EIS data reduction system and that using the GaBoDS pipeline (Schirmer et al. 2003; Erben et al. 2005) developed in Bonn were compared. Extensive and repeated comparisons were made between SExtractor-produced catalogs from the images generated by these two independent systems. Initial discrepancies, resulting from the different techniques used (e.g. cosmic ray removal, gain-harmonization) were resolved, leading at the end to results in excellent agreement.

A similar comparison has been partially carried out for the present dataset and it has shown an important short-coming of the current implementation of the EIS/MVM library. Even though the chip-to-chip gain correction is not mandatory, the log file created by the library provides no information of whether or not it has been applied. In fact, comparison of a subset of frames showed that in the original reductions made public late 2004 the images for DEEP2 were not homogenized. Since in the final, sky-subtracted image, residual differences are not seen this was only detected from the comparison with the independent reduction conducted in Bonn (see also Hildebrandt et al. 2006). It is important to emphasize that the images for DEEP2 have been rereduced and have replaced those released in October 2004. The same is true for the associated science-grade catalogs.

\subsection{Radio/optical correlation}

As mentioned earlier, the DEEP1 region was chosen to complement the sub-mJy ATESP $1.4 \mathrm{GHz}$ radio survey carried out with the Australia Telescope Compact Array (ATCA). The ATESP survey covers a strip of $26 \times 1$ square degrees, at declination $\delta \sim-40^{\circ}$, and has provided a catalogue of $\sim 3000$ radio sources to a flux limit of $S_{1.4 \mathrm{GHz}} \sim 0.4 \mathrm{mJy}$ (Prandoni et al. 2000a,b). The aim of the ATESP radio survey is to study the evolutionary properties of the objects belonging to the mJy and sub-mJy populations, by directly measuring the evolution of star-forming radio sources, in the redshift range $0<z<1$, and of lowluminosity AGNs up to higher redshifts.

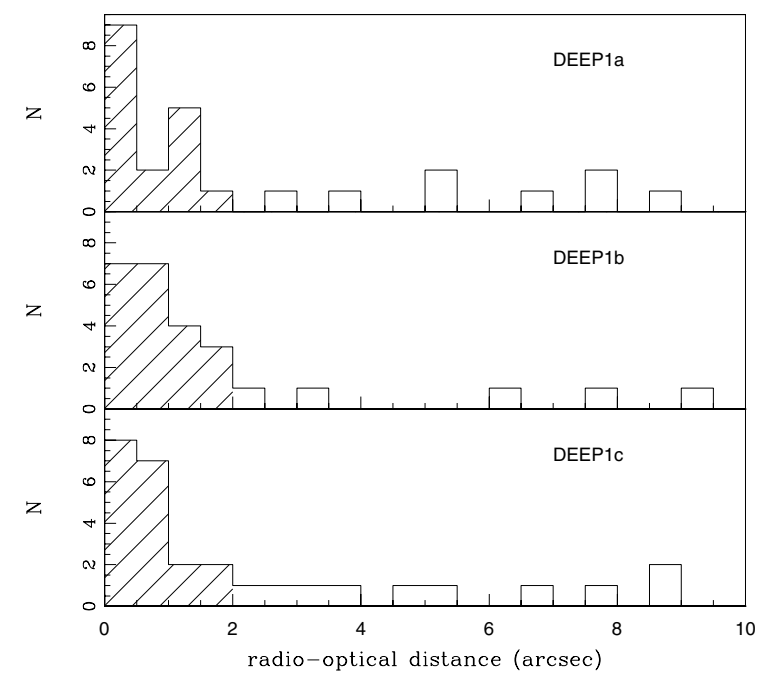

Fig. 2. Distribution of separations bewtween radio sources and the nearest $R$-detected sources for DEEP1a (upper panel), DEEP1b (middle panel) and DEEP1c (lower panel). The dashed histograms show those considered as actual counterparts.

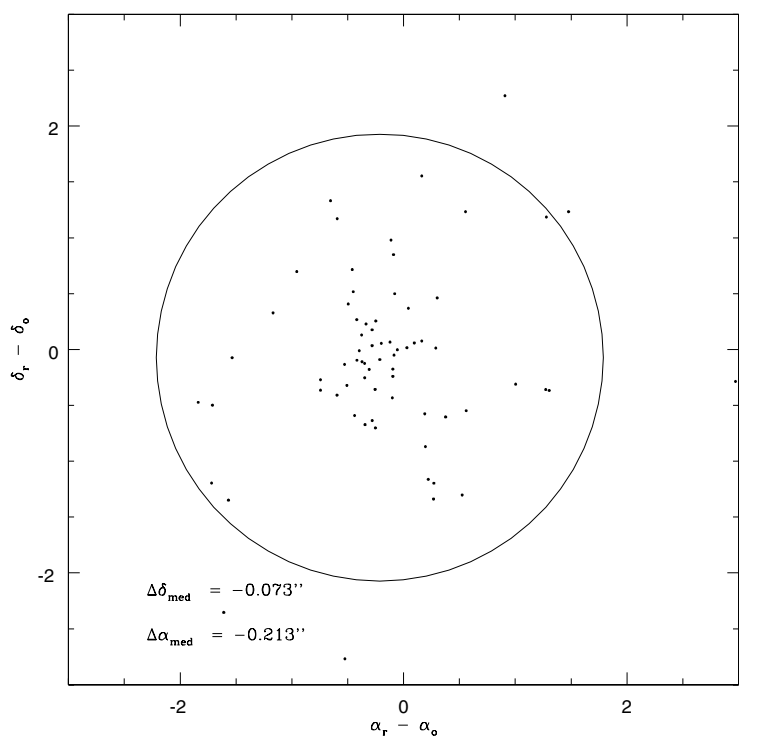

Fig. 3. Radio-optical offset computed for all the optical counterparts in the fields DEEP1 a,b,c. The 2 arcsec circle defines the boundaries of those considered to be "good" identifications. Also reported are the values of the median of the offset distribution in right ascension and declination.

In the $4 \times 0.25$ square degrees of the DEEP1 region covered by DPS there are 109 ATESP $1.4 \mathrm{GHz}$ radio sources - 25 in DEEP1a, 26 in DEEP1b, 29 in DEEP1c and 29 in DEEP1d. Since the latter field was not covered by the present survey, the analysis below refers to only 80 radio sources. To identify optical counterparts of these radio sources the nearest optical object was searched for. All the available single passband catalogs for the fields DEEP1a, b and c were treated separately. Figure 2 shows the radio-optical distance distribution of the $R$-selected optical counterparts of the ATESP radio sources for DEEP1a, b and c, respectively. As can be seen most optical counterparts are found within 2 arcsec. Beyond this distance, the distribution is flat, as expected for spurious identifications. Therefore, in this preliminary analysis this value is taken to be the maximum distance from a radio source for an $R$-band object to be considered its optical counterpart. The distribution of offsets for all the identified radio source-optical pairs is shown in Fig. 3. It is worth 


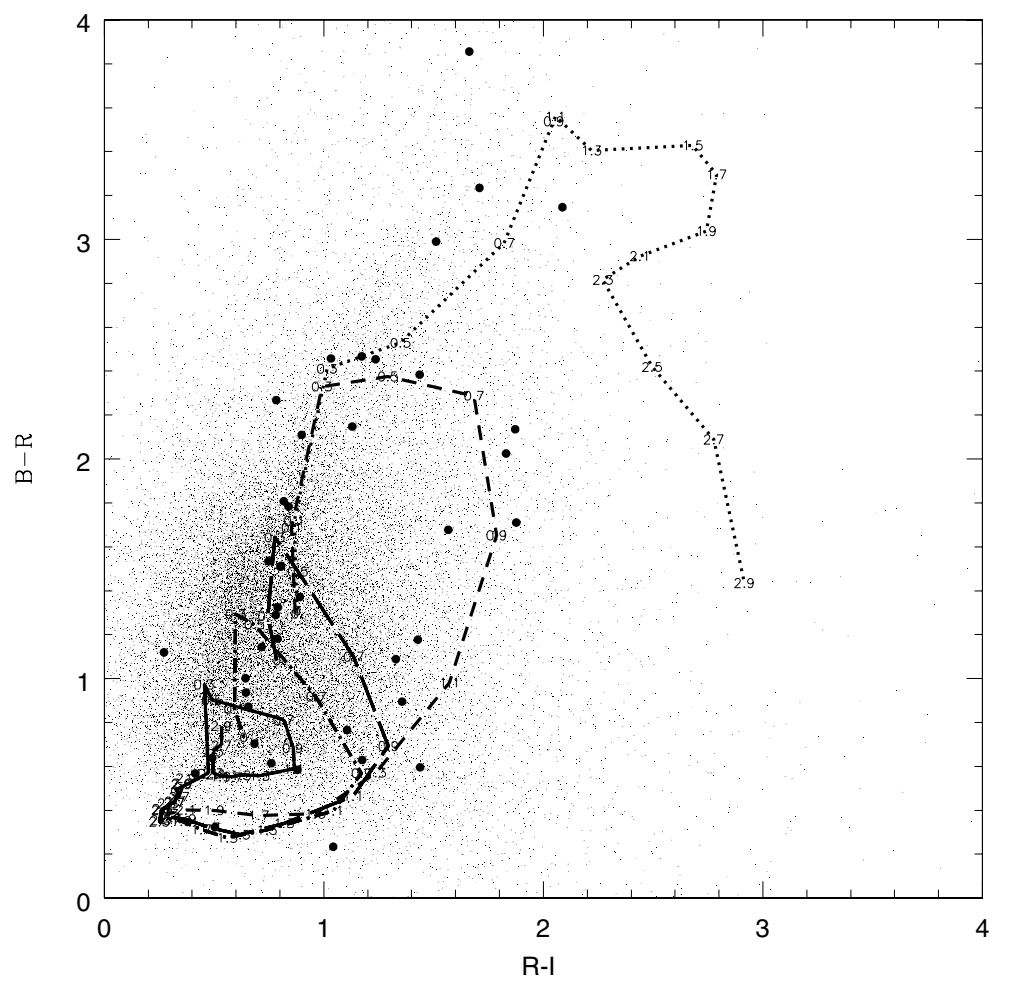

Fig. 4. Color-color diagram. In the figure is plotted the $(R-$ $I) \times(B-R)$ colors for the whole optical population (dots) and for the optical counterparts of ATESP radio sources (filled circles). Dot, dashed, long-dashed, dot-dashed and solid lines represent the evolutionary tracks of bursts (single burst of star formation followed by passive evolution), ellipticals, $\mathrm{Sb}$ and $\mathrm{Sc}$ spirals, and starburst galaxies, respectively. The evolutionary tracks were computed at intervals of 0.2 in redshift using the GISSEL libraries (Bruzual \& Charlot 1993). The model tracks span the redshift range $0.0<z<3.0$. noticing that systematic offsets in the relative position between the radio and optical sources are only -0.073 in declination and -0.213 in right ascension (see median values in Fig. 3), and have therefore been neglected. Also note that matches obtained in the $R$-band are the most complete, since all the radio sources identified in any of the $U_{-}, B-, V$ - or $I$-bands are also identified in $R$-band.

The ATESP source identification rate varies from $66 \%$ to $81 \%$, depending on the field, while the estimated contamination is $10-14 \%$. These identification rates are consistent with the value of $74 \%$ reported for the VVDS-VLA sample (Ciliegi et al. 2005), where a radio/optical analysis of a sample of $\mu \mathrm{Jy}$ sources was performed down to a similar optical depth. It is also interesting to compare these results to those obtained using a shallower survey, such as the $I$-band EIS-WIDE survey (Patch A, Nonino et al. 1999). In the 3 square degrees region that this patch covers, a radio/optical analysis was carried out, yielding the identification of $\sim 57 \%$ ATESP radio sources down to $I=22.5$, the limiting magnitude of the optical imaging (Prandoni et al. 2001). By comparing this identification rate to the one obtained with the DPS data, one finds that there is a significant increase, demonstrating the need for deep follow-up surveys for properly identifying the $\mathrm{mJy} / \mathrm{sub}-\mathrm{mJy}$ population.

The multi-band DPS data was used to analyze the color properties of the ATESP radio sources. Figure 4 shows in the $(R-I) \times(B-R)$ color diagram the location of the optical objects associated to the radio sources (filled circles), compared to the colors of field galaxies (dots). Also plotted are the evolutionary tracks of various optical galaxy populations (from early-type to spiral to starburst galaxies) in the redshift range $0<z<3$. From this plot it is clear that while the majority of the ATESP sources follows the early-type galaxy tracks (dotted and dashed lines) up to redshift $\sim 2$, some ATESP sources have colors consistent with being spiral or starburst galaxies (long-dashed, dot-dashed and solid lines) up to $z \sim 0.5-1$. These results are consistent with those of previous work (e.g. Prandoni et al. 2001) and the most likely interpretation is that classical radio galaxies, usually associated to early-type objects (ellipticals and/or S0) are, in general, more powerful and can be seen to larger redshifts than radio sources associated to star-forming galaxies.

To dramatically increase the identification rate of the ATESP sources, one requires deep near-infrared $(J, H, K)$ imaging. In this respect, the infrared part of DPS (see Olsen et al. 2006) is very useful, since it provides $J$ and $K$ images down to $K_{A B}=$ 21.3 for the DEEP1a and $b$ fields. Such data should allow the identification of the most distant radio galaxies (reddened by redshift effects) and possible "rare" intrinsically red objects (like EROs, ULIRGs, etc), probably reddened by dust.

The preliminary radio/optical analysis described above will be further developed in a future paper (Mignano et al. in prep.), where the multi-color information will be used to determine spectral types and (photometric) redshifts for the identified radio sources. We notice that, to fully exploit the capabilities of the optical data described above, the entire DEEP $12^{\circ} \times 0.5^{\circ}$ region has been imaged also at $5 \mathrm{GHz}$, again using the ATCA. The $5 \mathrm{GHz}$ data (Prandoni et al. 2006) give the possibility of measuring the radio spectral index (between $1.4 \mathrm{GHz}$ and $5 \mathrm{GHz}$ ) of the radio sources. This additional information may help constraining the origin (star formation or nuclear activity) of the radio emission in mJy and sub-mJy radio sources and in understanding whether it is linked to the optical light.

\section{Summary}

This paper presents the survey products prepared by the EIS team for the optical part of the Deep Public Survey (DPS) conducted over 4 years by the team. The survey was designed to cover 3 independent strips of the sky with an area of 1 square degree each in five passbands. The paper describes the observations and presents a comprehensive discussion of the set of products prepared and validated by the EIS Data Reduction System. These products are publicly available including fully calibrated 
reduced images taken on a nightly basis, final stacked images and extracted source catalogs. It should be noted that the present optical data are complemented by observations in infrared reported by Olsen et al. (2006).

Acknowledgements. We would like to express our thanks to several other EIS visitors who have contributed directly or indirectly to the results presented in this paper, too numerous to list individually. Special thanks to L. Girardi for providing us with the means to derive the color-color tracks used in this paper, which were created for the filters used by EIS. L.F.O. acknowledges financial support from the Carlsberg Foundation, the Danish Natural Science Research Council and the Poincare fellowship program at the Observatoire de la Côte d'Azur. The Dark Cosmology Centre is funded by the Danish National Research Foundation.

\section{References}

Arnouts, S., Vandame, B., Benoist, C., et al. 2001, A\&A, 379, 740 Bertin, E., \& Arnouts, S. 1996, A\&AS, 117, 393

Bruzual, G., \& Charlot, S. 1993, ApJ, 405, 538
Ciliegi, P., Zamorani, G., Bondi, M., et al. 2005, A\&A, 441, 879

Dietrich, J. P., Miralles, J.-M., Olsen, L. F., et al. 2006, A\&A, 449, 837

Erben, T., Schirmer, M., Dietrich, J. P., et al. 2005, Astron. Nachr., 326, 432

Girardi, L., Groenewegen, M., Hatziminaoglou, E., \& da Costa, L. 2005, A\&A, 436, 895

Hildebrandt, H., Erben, T., Dietrich, et al. 2006 A\&A, 452, 1121

Landolt, A. U. 1992, AJ, 104, 340

Metcalfe, N., Shanks, T., Campos, A., McCracken, H. J., \& Fong, R. 2001, MNRAS, 323, 795

Nonino, M., Bertin, E., da Costa, L., et al. 1999, A\&AS, 137, 51

Olsen, L. F., Miralles, J.-M., da Costa, L., et al. 2006, A\&A, 456, 881

Prandoni, I., Gregorini, L., Parma, P., et al. 2000a, A\&AS, 146, 31

Prandoni, I., Gregorini, L., Parma, P., et al. 2000b, A\&AS, 146, 41

Prandoni, I., Gregorini, L., Parma, P., et al. 2001, A\&A, 369, 787

Prandoni, I., Parma, P., \& Weiringa et al., M. H. 2006, A\&A, 457, 517

Renzini, A., \& da Costa, L. 1997, The Messenger, 87, 23

Schirmer, M., Erben, T., Schneider, P., et al. 2003, A\&A, 407, 869

Schlegel, D., Finkbeiner, D., \& Davis, M. 1998, ApJ, 500, 525

Vandame, B. 2004, Ph.D. Thesis, University of Nice-Sophia Antipolis

Vettolani, G., Zucca, E., Zamorani, G., et al. 1997, A\&A, 325, 954 
A. Mignano et al.: ESO imaging survey: optical deep public survey, Online Material $p 1$

\section{Online Material}


A. Mignano et al.: ESO imaging survey: optical deep public survey, Online Material $p 2$

\section{Appendix A: Galaxy Counts}

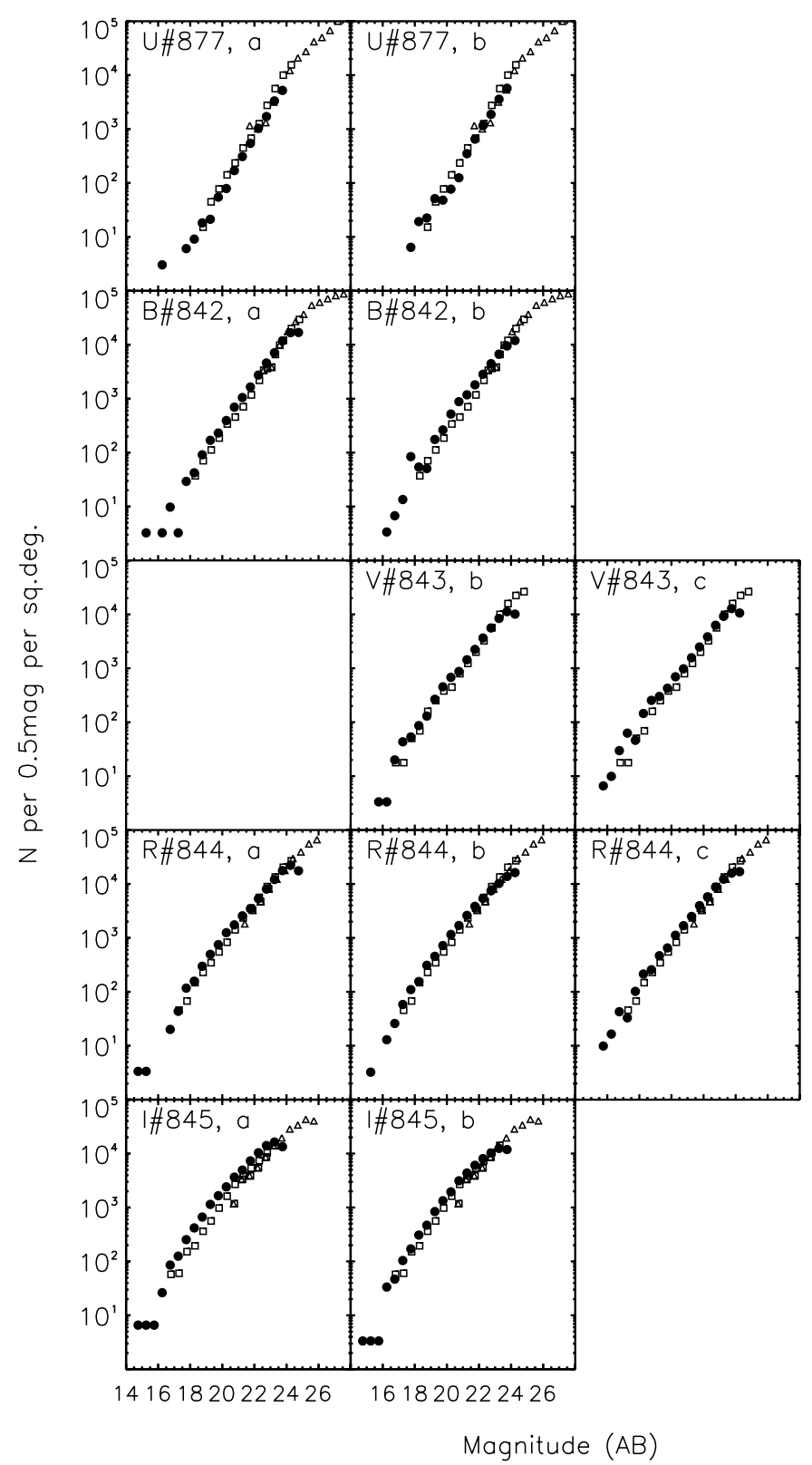

Fig. A.1. Galaxy number counts for the fields in the DEEP1 region (solid points) compared to those of Arnouts et al. (2001) (open squares) and Metcalfe et al. (2001) (open triangles). The passband, filter identification number and field are indicated in each panel. 
A. Mignano et al.: ESO imaging survey: optical deep public survey, Online Material $p 3$

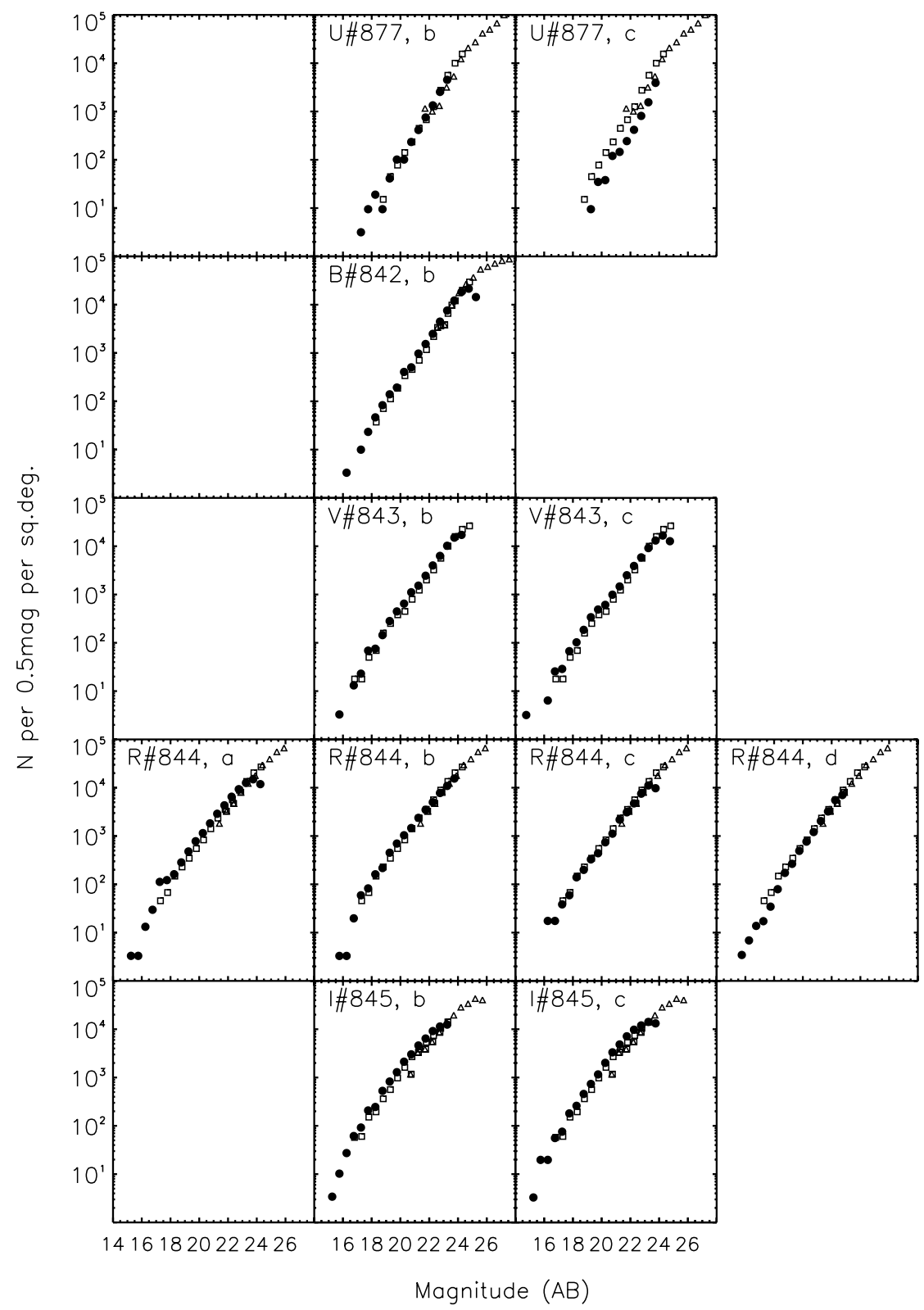

Fig. A.2. Same as in Fig. A.1 for region Deep2. 
A. Mignano et al.: ESO imaging survey: optical deep public survey, Online Material p 4

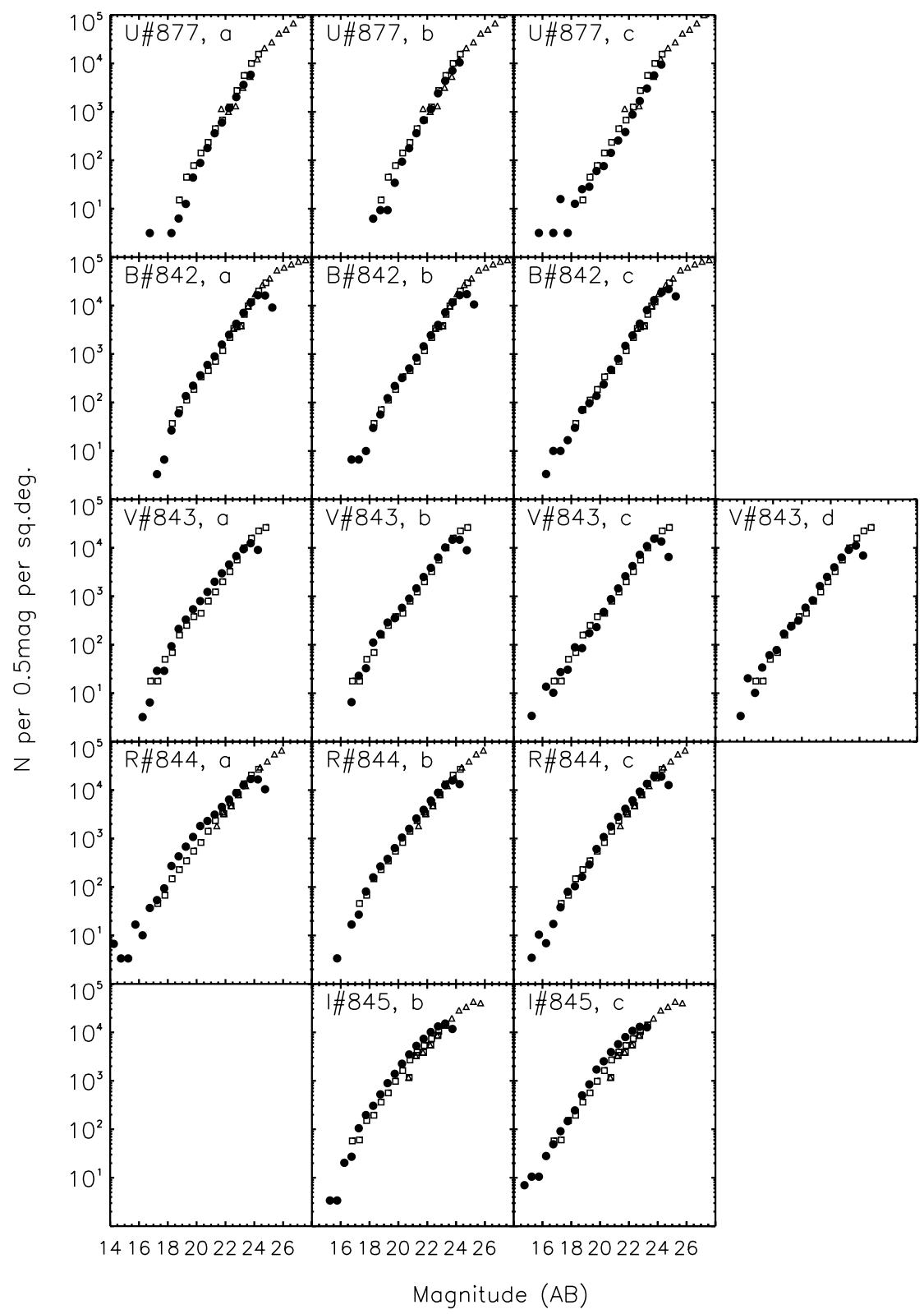

Fig. A.3. Same as in Fig. A.1 for region Deep3. 
A. Mignano et al.: ESO imaging survey: optical deep public survey, Online Material p 5

\section{Appendix B: Stellar Counts}

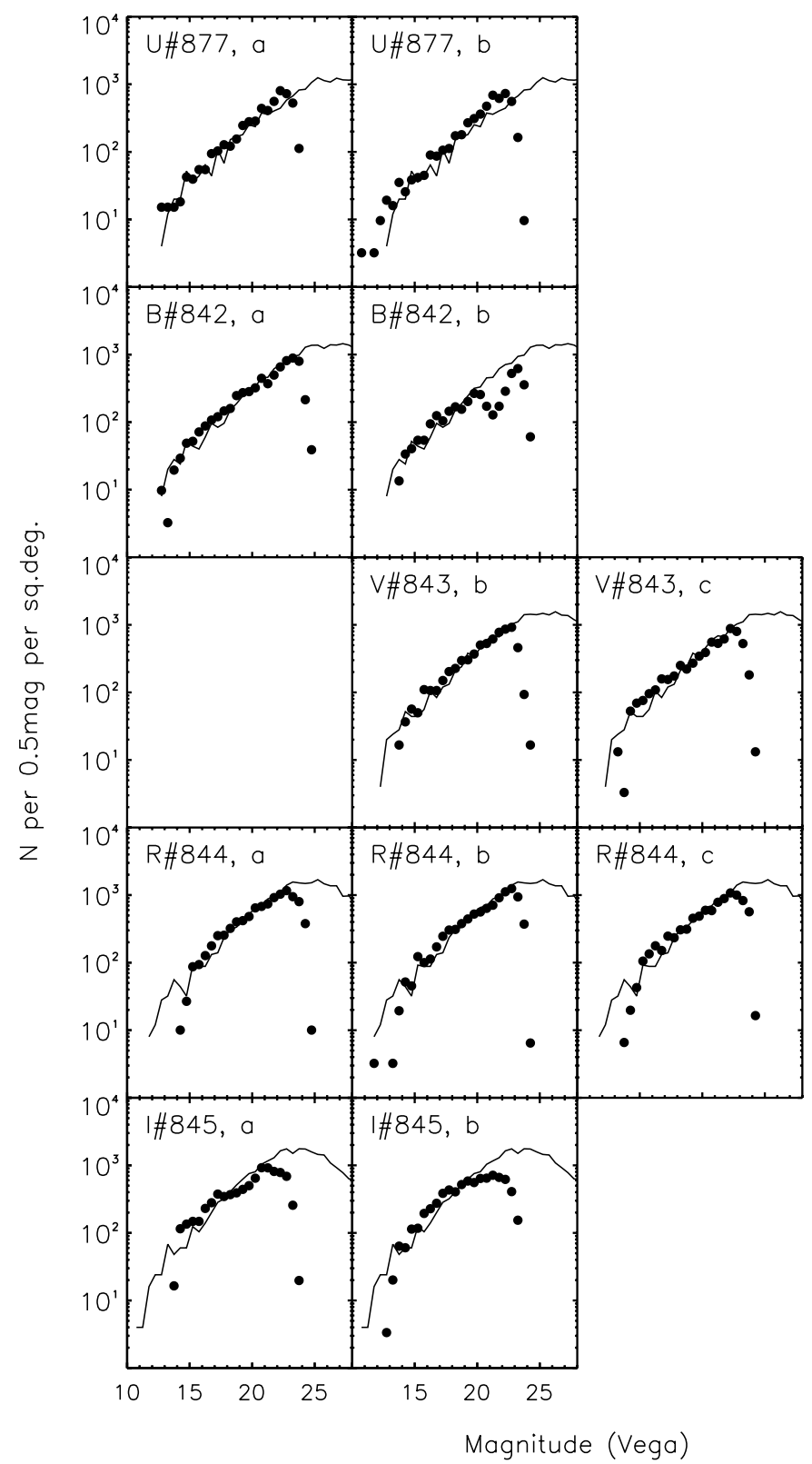

Fig. B.1. Stellar number counts (black points) compared with model predictions (solid line) from Girardi et al. (2005). The passband, filter identification number and field are indicated in each panel. 
A. Mignano et al.: ESO imaging survey: optical deep public survey, Online Material p 6

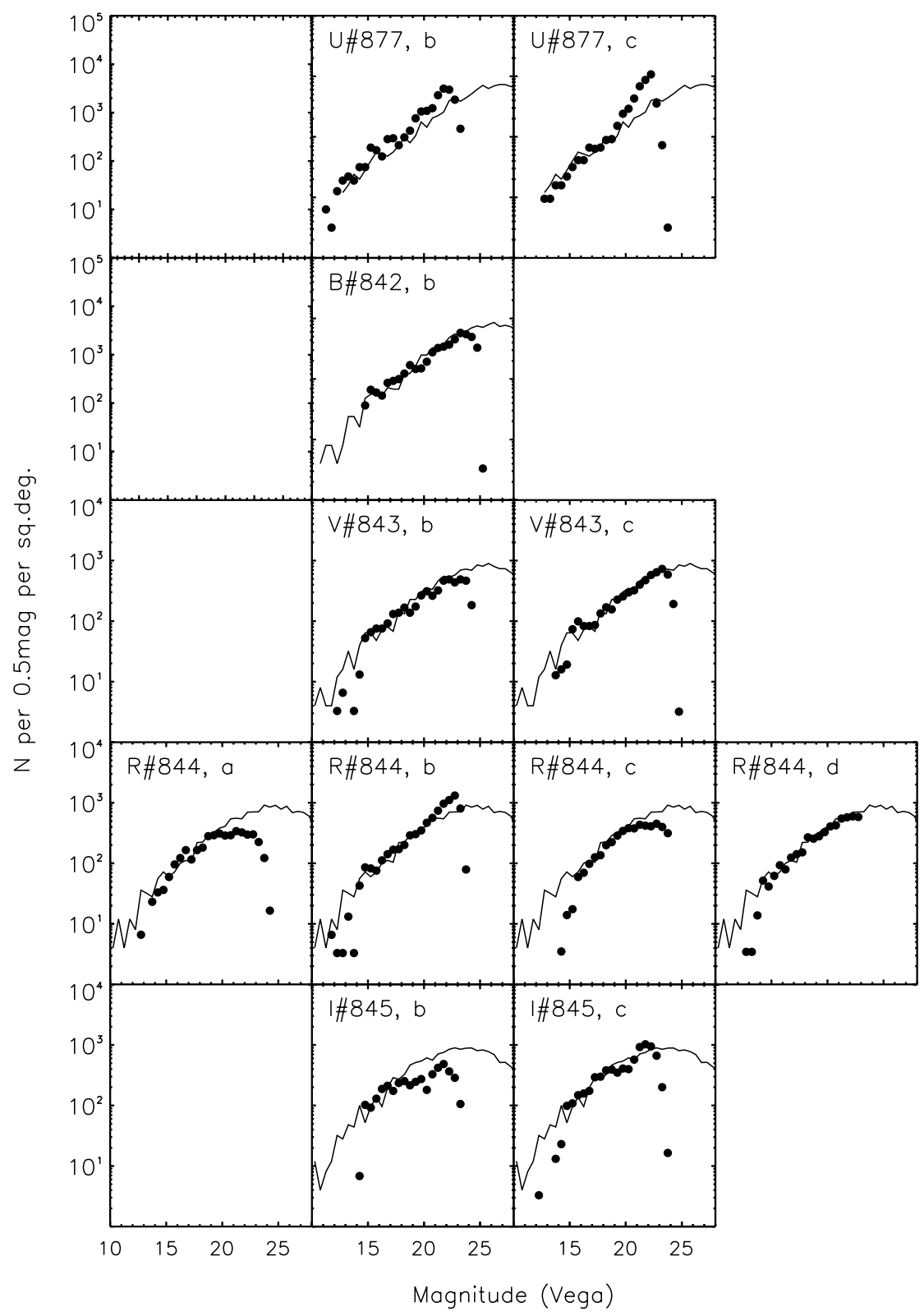

Fig. B.2. Same as Fig. B.1 but for the DEEP2 region. 
A. Mignano et al.: ESO imaging survey: optical deep public survey, Online Material $p 7$

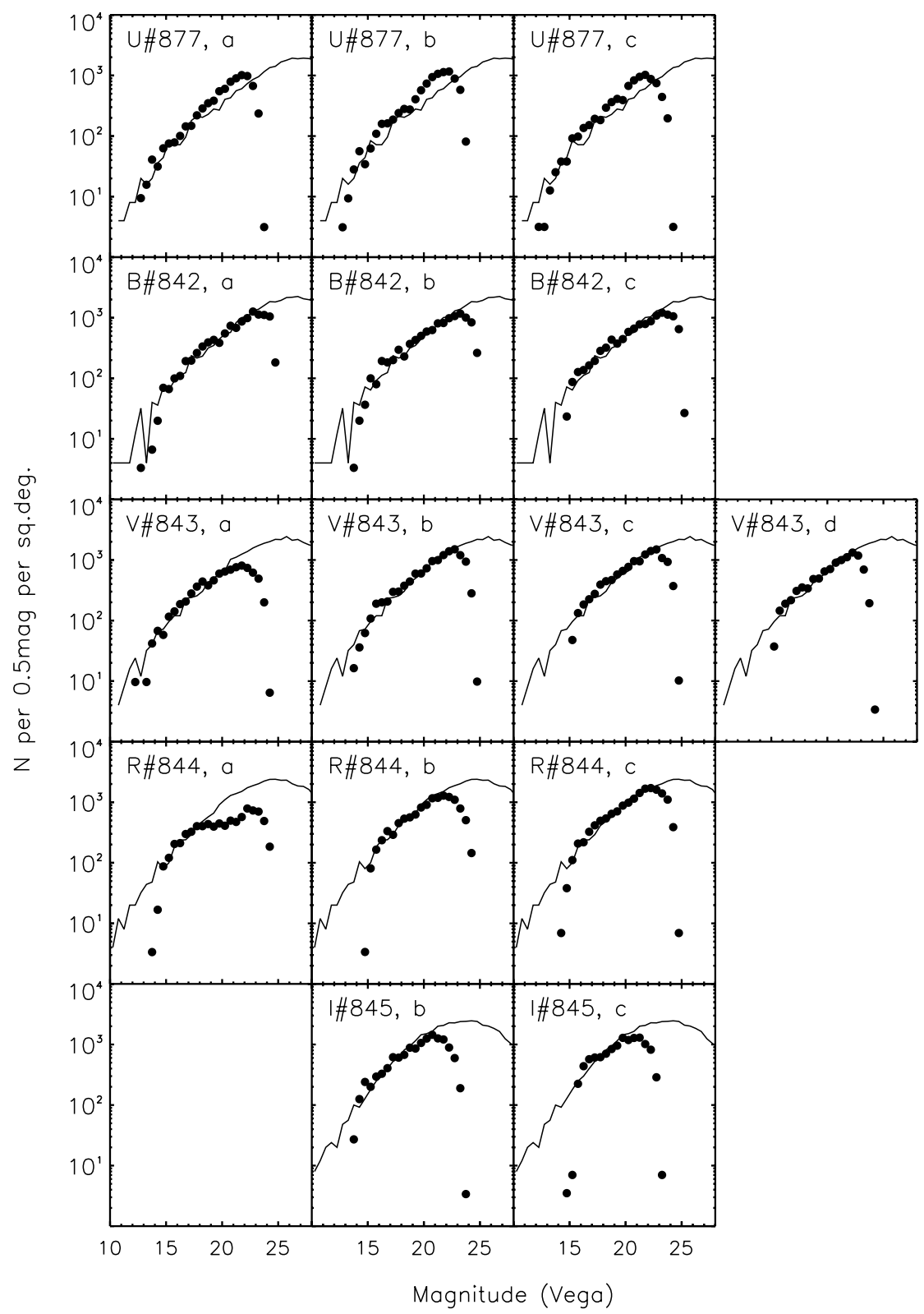

Fig. B.3. Same as Fig. B.1 but for the DEEP3 region. 
A. Mignano et al.: ESO imaging survey: optical deep public survey, Online Material $p 8$

\section{Appendix C: Color-color Diagrams}
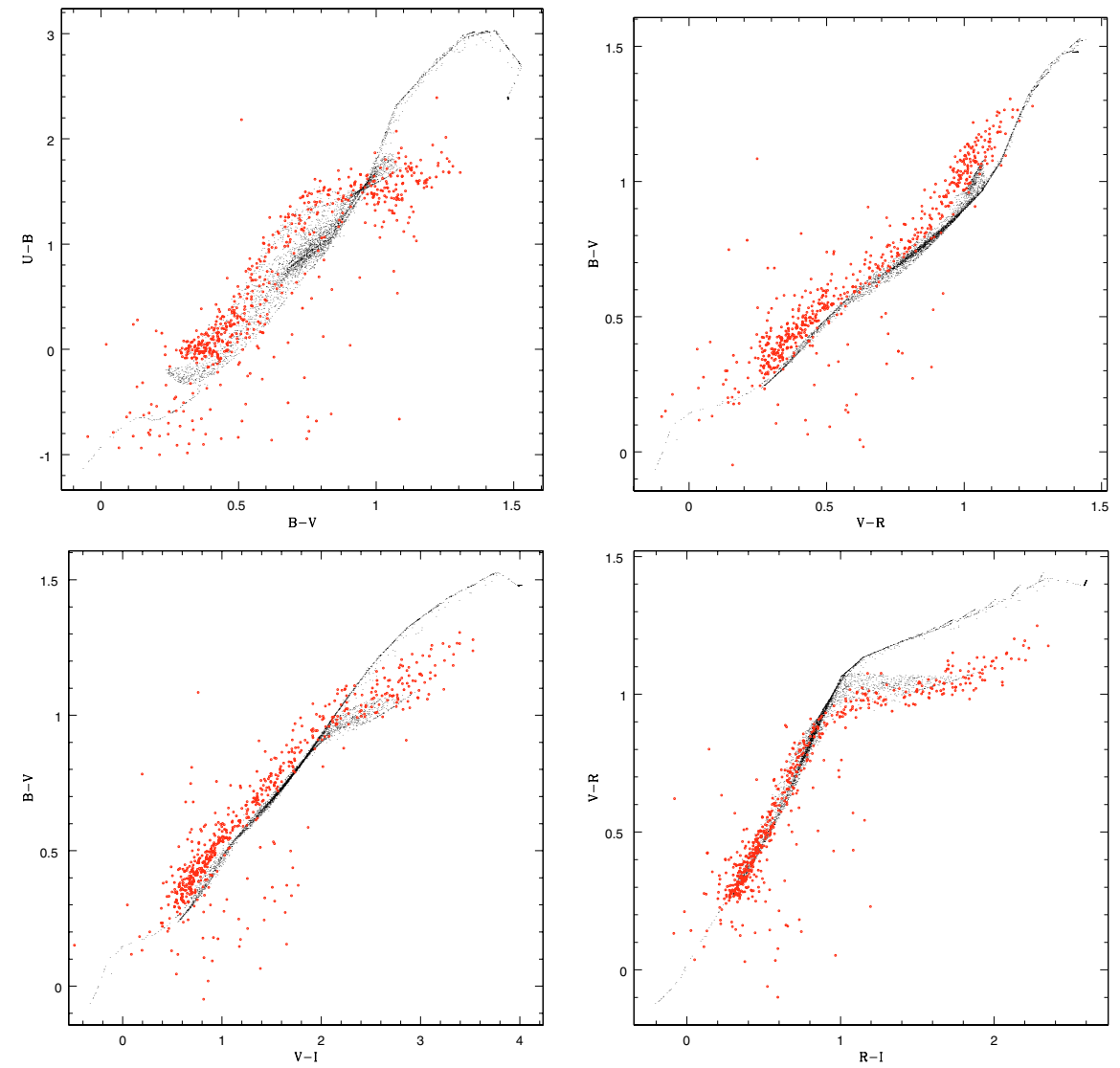

Fig. C.1. Color-color plot for stars (red points) selected in the field DEEP1b compared the model of Girardi et al. (2005) (black points). 
A. Mignano et al.: ESO imaging survey: optical deep public survey, Online Material $p 9$
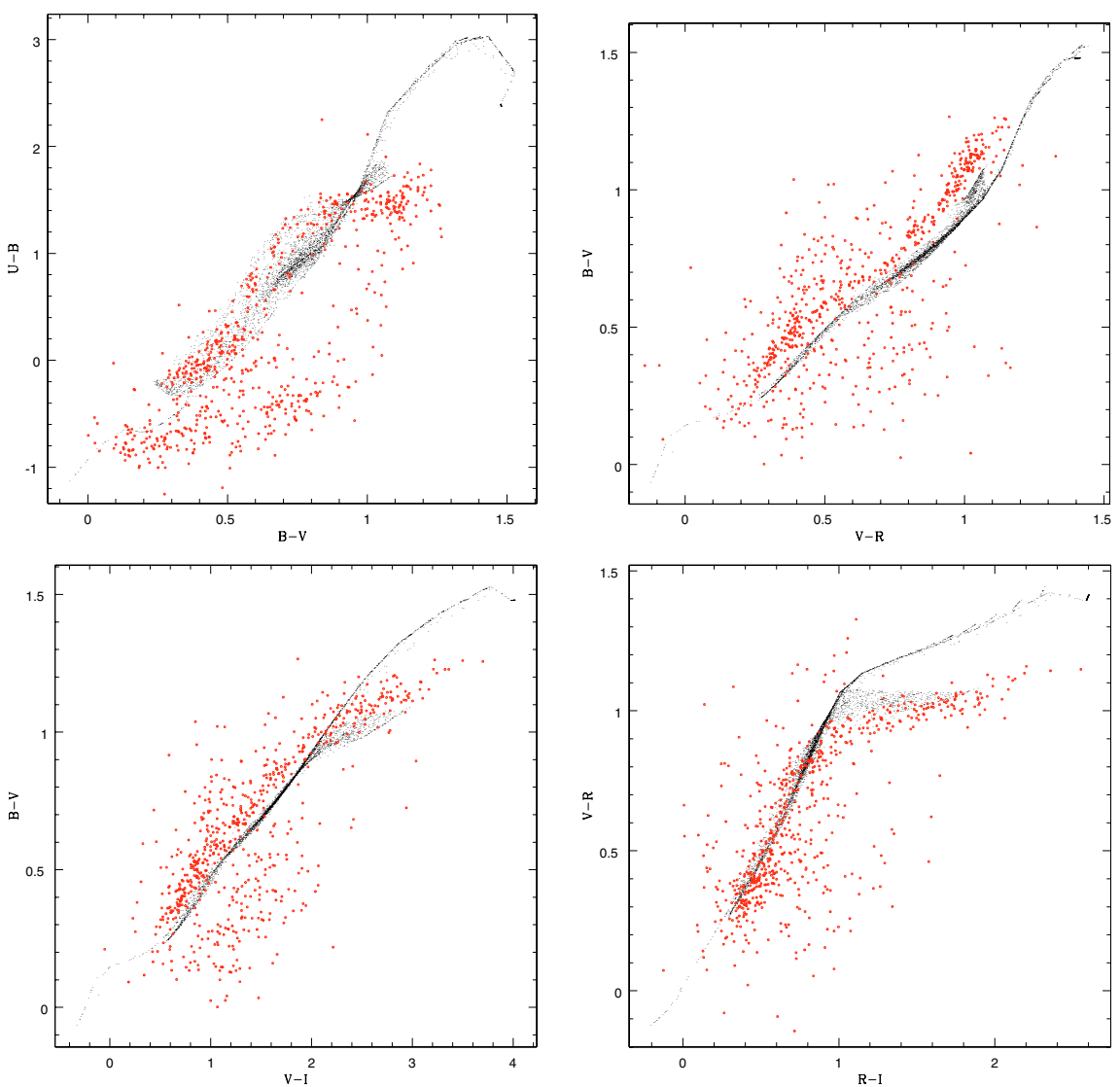

Fig. C.2. Same as Fig. C.1 but for field DEEP2b.
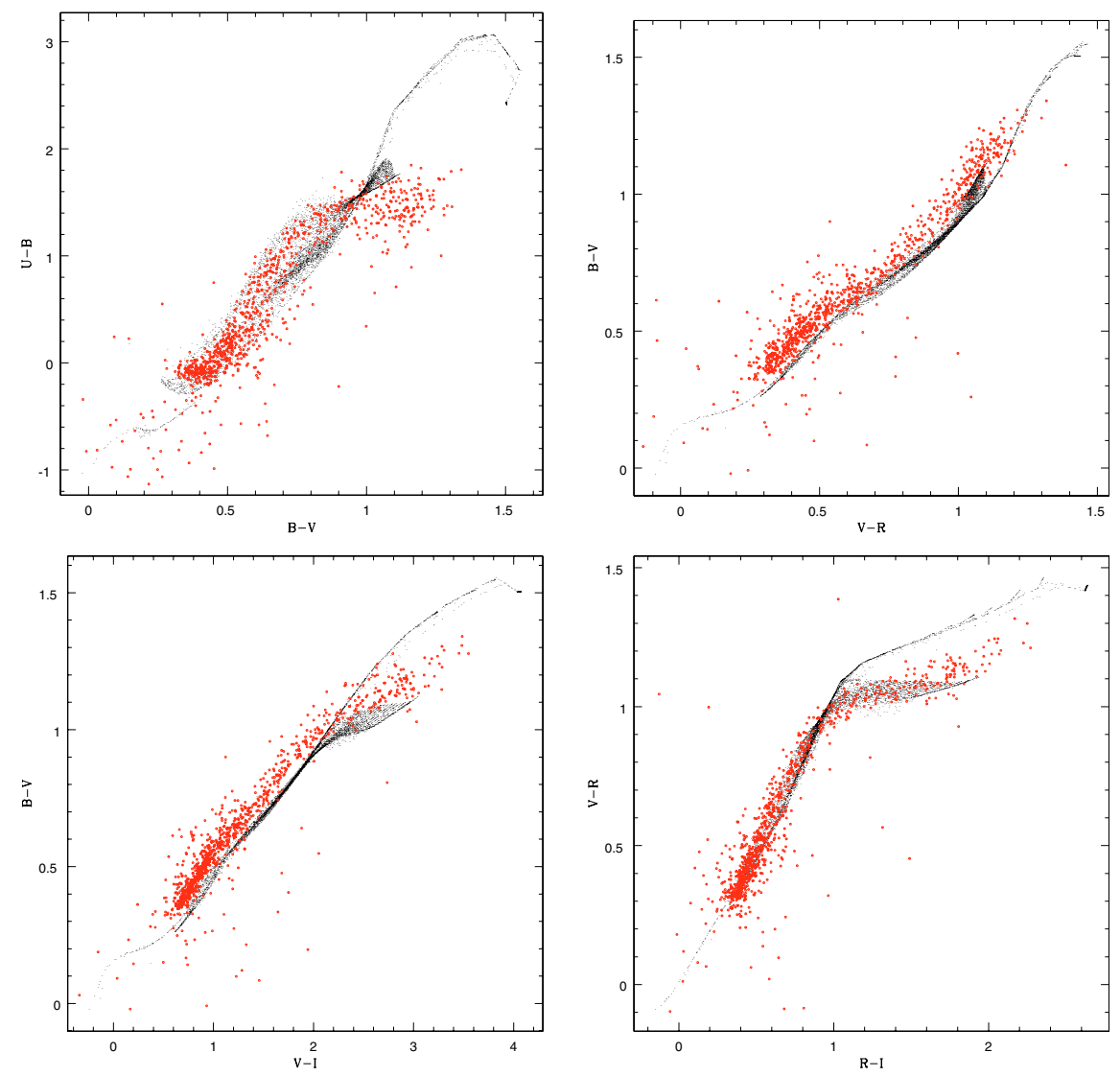

Fig. C.3. Same as Fig. C.1 but for field DEEP3b. 
A. Mignano et al.: ESO imaging survey: optical deep public survey, Online Material p 10
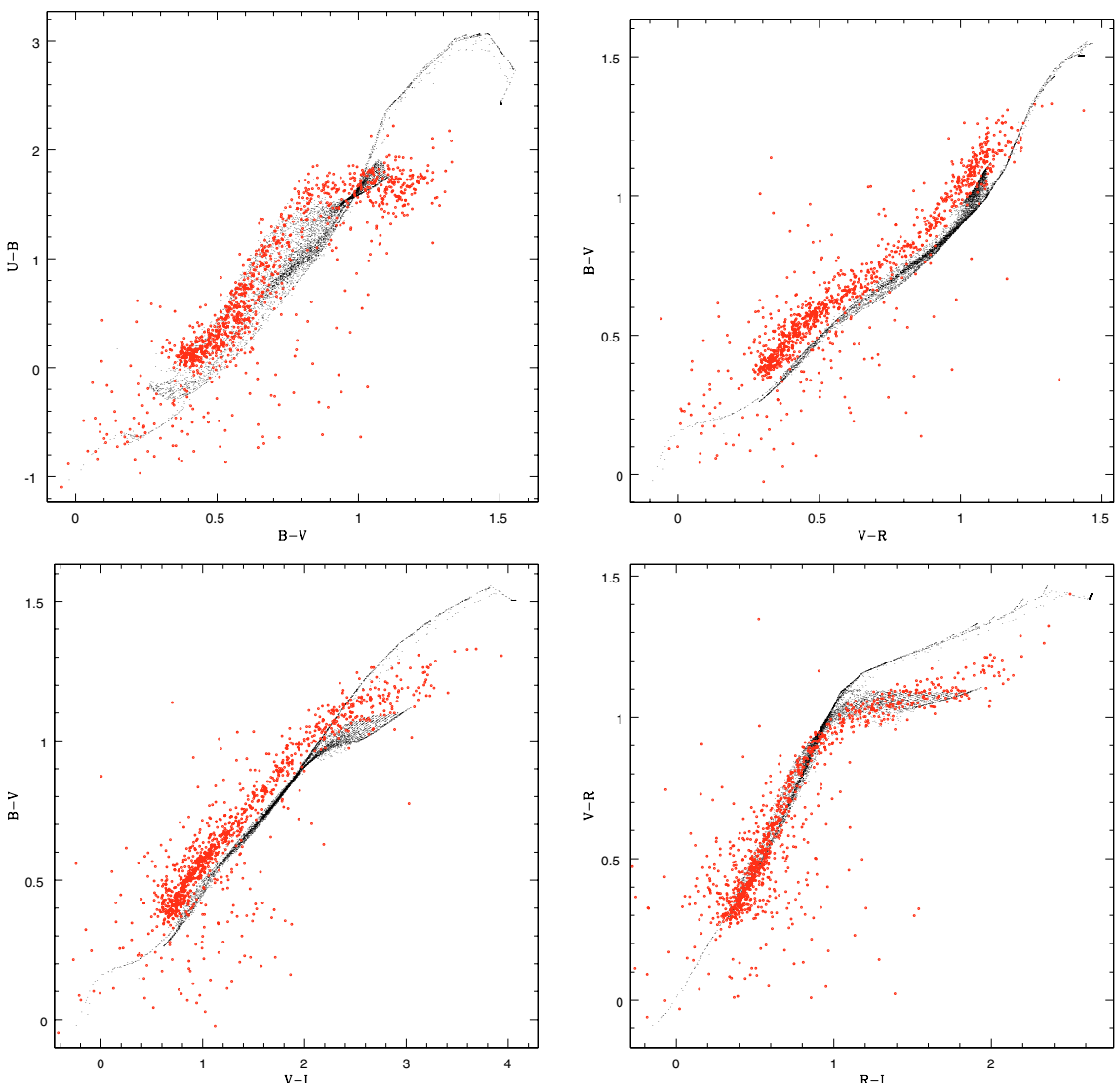

Fig. C.4. Same as Fig. C.1 but for field DEEP3c. 